\title{
The Angle Trisection Solution (A Compass-Straightedge (Ruler) Construction)
}

\author{
Kimuya .M. Alex ${ }^{1}$, Josephine Mutembei² \\ ${ }^{1}$ Cell; +254 $704600418{ }^{2}$ Cell; +254 721567967 \\ 1alexkimuya23@gmail.com \\ 2joymutembei23@gmail.com
}

${ }^{1}$ Meru University of Science and Technology, Kenya, Department of Physical Sciences, Faculty of Physics

${ }^{2}$ Meru University of Science and Technology, Kenya, Department of Mathematics

\begin{abstract}
This paper is devoted to exposition of a provable classical solution for the ancient Greek's classical geometric problem of angle trisection [3]. (Pierre Laurent Wantzel, 1837), presented an algebraic proof based on ideas from Galois theory showing that, the angle trisection solution correspond to an implicit solution of the cubic equation; $x^{3}-3 x-1=0$, which he stated as geometrically irreducible [23]. The primary objective of this novel work is to show the possibility to solve the ages old problem of trisecting an arbitrary angle using the traditional Greek's tools of geometry (classical compass and straightedge), and refute the presented proof of angle trisection impossibility statement. The exposed proof of the solution is theorem 4.0 , which is based on the classical rules of Euclidean geometry, contrary to the Archimedes proposition of using a marked straightedge construction [4], [11].
\end{abstract}

\section{Key Words}

Angle trisection; An arbitrary angle; A particular angle; Compass; Ruler (Straightedge); Classical geometry; GeoGebra Software; Plane geometry; Subset; Superset; Singular angle

\section{Notations}

$\begin{array}{ll}\angle & \text { Notation for an angle } \\ \overline{\cap \forall} & \text { Denotes a straight line segment and a length } \\ 2 D & \text { Two Dimensions } \\ 3 D & \text { Three Dimensions } \\ 4 s . f & \text { Four significant figures } \\ \in & \text { Denotes a member or an element of a particular set } \\ \subset & \text { Subset } \\ \notin & \text { Not a member or an element of a particular set } \\ a \backslash b & \text { a divides } b \\ \cos \theta & \text { Cosine of angle } \theta \\ 5 d p & 5 \text { decimal places }\end{array}$

\section{Academic Discipline And Sub-Disciplines}

Mathematics (Geometry)

\subsection{INTRODUCTION}

Compass and straightedge problems have always been the favorite subject of classical geometry. There are three classical problems of geometry posed by the ancient Greek geometers which include; "Trisection of an arbitrary angle", "Squaring of a circle", and "The duplication of a cube". Through the ages, mathematicians have expe nded a vast amounts of energy in efforts to find solutions for the three problems, but no geometrical solutions have been discovered by this day. Though the three problems are closely related, the focus of this work is to separately provide an elegant solu tion for the problem of angle trisection. The problem of angle trisection concerns the classical partitioning of a given angle into three mean proportions using the traditional Greek's tools of geometry (classical compass and straightedge). In 19th century, 
(Pierre Laurent Wantzel, 1837) presented an abstruse algebraic proof bas ed on concepts from Galois theory showing that, the solution of the angle trisection problem corresponds to solution of the cubic equation; $x^{3}-3 x-1=0$, which is algebraicallyirreducible [2], and therefore it cannot be redressed using the Greek's tools of geometry. This proof pose a serious geometrical misconception in the sense that, it does not take into account, the conditions governing solutions to the three stated geometrical problems of antiquity. Study results show that by the time the angle trisection impossibility proof was stated, there alreadyexisted so manytheorems in geometry, and it was clear there was a need to systematize and organize all of the material into a coherent, logical whole. The study of geometry transitioned from a practical science to a more general, abstract view of relationships in an ideal existence [21]. One of the major conditions establis hed as part of this transition was that plane geom etric constructions must be carried out solely with a straightedge and compass, since specifically, a straightedge can be used in drawing a straight line between two given points, and that a circle with a given radius can be drawn around a given point using a compass (application of the compass equivalence theorem). Any other form of construction not using the compass and straightedge is strictlyprohibited [19]. This pres ent work is confined in the specified restrictions of Euclidean geometry. The impossibility avowal of angle trisection made mathematicians completely, wick into mechanical methods of solving the trisection of a given angle. But, however, despite bending the set rules governing plane geometry, none of the presented methodologies is absolutelycorrect. The deeper desire by mathematicians and other practitioners to have the angle trisection problem sought concerns serious misinterpretations of the problem, as evident in [1], [3], and [8], that the genetic definition of angle trisection problem is still not well understood. Clearly, the trisection of a particular angle does not mean the possibility to solve the trisection of a $n$ arbitrary angle. Section 1.1 provides a clear discussion, to define the angle trisection problem under the set conditions of Greek's geometry; to bring out the geometrical interpretation of the angle trisection problem and reveal why all the presented methods aimed at the angle trisection solutions are generallyincorrect. Section 1.3 presents two interp reted versions of the angle tris ection impossibility proof, to show the geometrical limitation of abstract algebra in its extension to Euclidean geometric proofs. Theorem 4 reveals the general geometric proof for the solution of an arbitrary angle trisection, and so is the trisection of a particular angle; while theorem 5 explains the classical trisection of both $0^{\circ}$ and $360^{\circ}$ angles. The most significant consideration in the revealed proof is to provide the basic necessary knowledge of trisecting any given angle (an angle defined between two rays) using onlycompass and straightedge. This conside ration is in harmony with the philosophical concern that, all geometric proofs should be elegant to follow, for different practitioners and everyone with interest in studying geometry, from the middle secondary school levels to graduate levels at universities. The difference between classical geometric and modern geometric semantic is especially conspicu ous in the case of quantities such as area and volume. As seen in Euclid's Elements there is no real number measure of the area of a plane figure. Instead, equality of plane figures is verified by cutting in pieces and adding and subtracting congruent triangles using the Pythagorean theorem (Book I of Euclid Elements, Proposition 47), which asserts "the squares on the sides of a right triangle, taken together, have the same content as the square on the hypotenuse (Longes $t$ side)". Thus in classical geometry, the term irrationality did not exist. The modern language of rational numbers was represented by a ratios of geometrical quantities based on positive integers. Any other quantity was represented as a geometrical magnitude. In accordance with Book III of La Géométrie [7], Descartes gave an account on the roots of cubic and quartic equations. He considers polynomials with integer coefficients. He put it that, if there is an integer root, that gives a numerical solution to the problem. But, if there are no integral roots, the solutions must be constructed geometrically. The proposed algorithm in this work is based on these traditional considerations. However, it com pletely does not omit the algebraic view of geometry in the modern world. An analytical interpretation is presented for every geometric proof, accompanied by the use of a computer software (GeoGebra) for results visualization, to bring the connection between the classical geometric approach and the computer aided design (CAD) methods.

\subsection{Classical definition of the Angle Trisection Problem}

This section, in brief, discuss the trisection of an angle based on the general understanding of an arbitrary angle. The problem requires one to construct an angle one third the size of a given angle, using onlytwo tools (Straightedge (ruler) and a compass). This definition pose two serious restrictions; that, the size of the given angle should notbe determined or measured, nor should it be a matter at all for any trisection construction (except for the singular angles $0^{\circ}$ and $360^{\circ}$ ), and that, any algorithm aimed at solving the trisection of an arbitrary angle should not involve arithmetic, but it s hould be typically geometrical. Figure 1 illustrates an example of an arbitrary angle on a plane. 


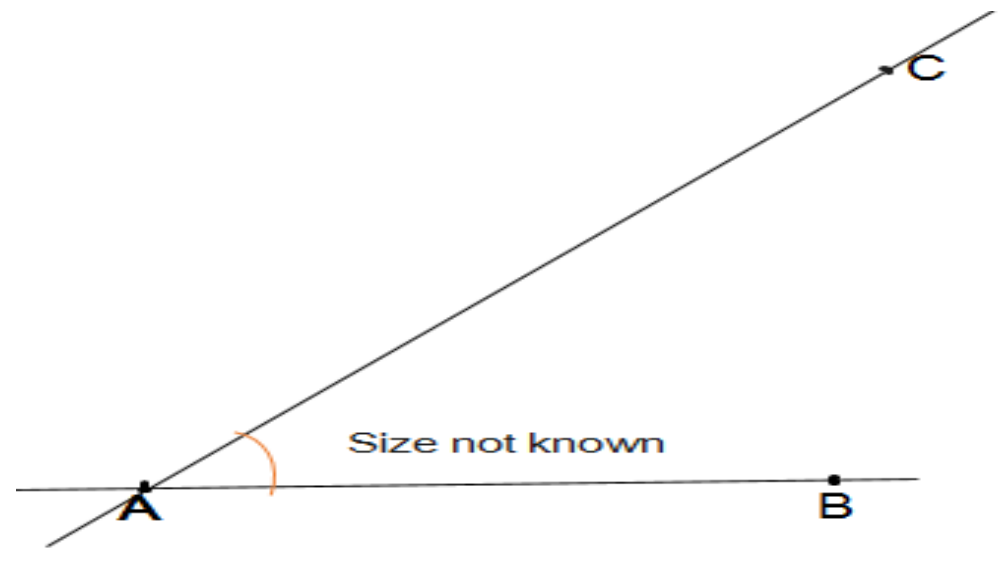

Fig 1: Representation of an arbitrary angle in plane geometry

\subsection{To show that for any three points one not lying on a straight line with the other two, the three points lie on the same plane}

The goal of this section is to elaborate on a proposition defined to expose that, any three points, one not collinear with the other two produce a plane geometric figure and that; no two such plane figures can share all the three vertices in common. For instance, a union of two rays sharing a common endpoint on a plane produce an angle of some size as illustrated in figure (1), $(\angle C A B)$. An example is in a geometrical figure such as a quadrilateral and a triangle. Any two sides of these objects share a common endpoint called a vertex and thus an angle of some size is defined between the two sides of the figure. Consider the following theorem:

Theorem 1: For any three points, one not lying in a straight line with the other two, the three points lie only on same plane, and that every triangle lies only on one plane [9].

Considering triangles $A B C$ and $A B D$ on planes $M$ and $N$ respectively, it is not practically possible for the two triangles to lie in both plane $M$ and in plane $N$, and so is their respective vertices as shown in figure (2). To verify this proposition, let points $A, B$, and $C$ lie on the two distinctplanes; $M$ and $N$, such that point $D$ is an image of point $C$. Since both point $A$ and $B$ lie on plane $M$, the straight line $A B$ lie on plane $M$. Also, since points $A$ and $B$ lie on plane $N$, the straight line $A B$ also lie on plane $N$. Therefore plane $M$ and plane $N$ have line $A B$ in common (theyintersect along line $A B$ ). Point $C$ does not lie in line with points $A$ and $B$, and therefore not common for both planes, a similar case for point $D$. Thus it is not geneticallypossible for points $A, B, C$ to lie on both planes $M$ and $N$. Likewise, it is not possible for poin ts $A, B, D$ to lie on both planes. Hence, for any plane containing all of triangle $A B C$ must also contain its three vertices $A, B$, and C. This shows that the whole of any triangle lies only in one plane. Considering either $\angle C A B$ or $\angle D A B$ as the acute angle to be trisected, it is required that all the points defining the "trisection angles" be defined in a two dimensional system (plane geometry). Thus the problem of trisecting an angle in general has to be sought following the classical rules of Euclidean geometry, and not using the mechanical methods being employed [10].

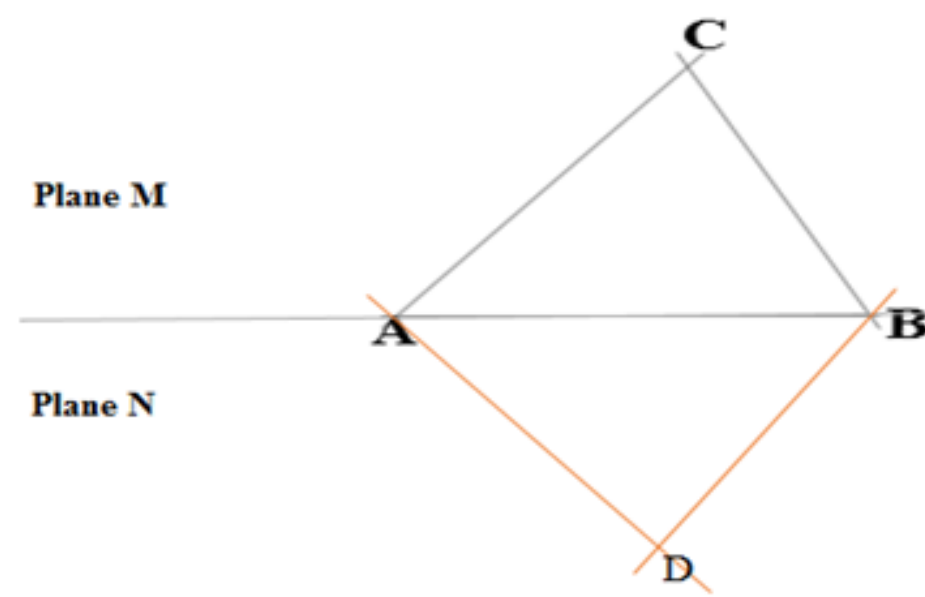




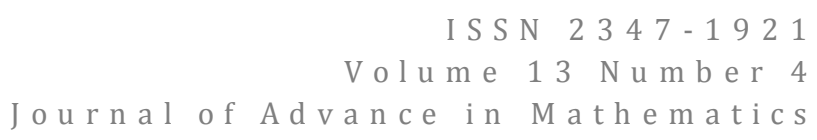

Fig 2: Illustration of a plane geometric figure

\subsection{The Mistake in the Angle Trisection Impossibility Algebraic Proof of (1837)}

Theorem 1 illustrates how three points defining a given angle, and one not collinear with the other two lie on the same plane, and that in plane geometry two different plane figures cannot share all points in common. This section stems from theorem 1, objectively to show the algebraic limitations in the presented proof of angle trisection impossibility statem ent, to explain the degree of misconception governing the methods meant as solutions for the angle trisection problem; to expose why the statement of angle trisection impossibility is not valid, and it thus should not be extended to plane geometric problems. For instance, consider the algebraic conditions that: a length is constructible if and on ly if it represents a constructible number, an angle is constructible if and only if its cosine is a constructible number, and that, a number is constructible if and only if it can be written in the four basic arithmetic operations $\left(t_{3},{ }_{3} X_{3} \div\right)$ and the extraction of the square roots but not on higher roots [22].These three conditions were greatly considered in generating the angle trisection impossibility, which has limited and discouraged researchers from exploring on the already exis ting realm of Greek's geometry, in their freedom to add value to Euclidean geometry [11]. Moreover, it has been proven that there are infinitely many angles that are geometrically cons tructible and trisectible [1]. It is possible to bisect both an a ngle and a straight line, as well as dividing a straight line segment into the desired number of equal portions. It is also possible to construct lines of magnitude $\sqrt{2}$ and $\sqrt{5}$, as well as constructing squares of contents (areas) 2 and 5 units. The existence of these well justified constructions show the probability to construct the algebraically declared irrational numbers, and so is trisection of a given angle, or the possibility of partitioning an angle into a certain ratio. According to this paper, the above stated algebraic constraints are not fashionable in restricting researcher from exploring the broad field of Euclidean geometry, since logically, geometry concerns following certain lines of reasoning, and the verification of the logic based on the obtained results is what matters. P.L.Wantzel's proof greatly depended on these algebraic conditions, and concepts from abstract algebra based on cubic extensions, to proof the algebraic inability to extract the roots of the cubic equation; $x^{3}-3 x-1=0$. Consider the following two versions of the impos sibility proof:

\subsubsection{An interpreted Version of the Angle Trisection Impossibility Proof based on a Lemma;} there is no power of $\mathbf{2}$ that is evenly divisible by $\mathbf{3}$

This lemma can be used to demonstrate the angle trisection impossibility using concepts from Galois field of numbers as stated in [1]. To improve on the dimensionality perspective as discussed in [1], a proof upon this lemma is des cribed as follows:

Corollary: Let $S$ be a field, and let $T$ be an extension of $S$ that is constructible out of $T$ by a finite order of quadratic extensions. Then $S$ does not contain any cubic extensions $U$ of $T$.

Proof: If $S$ contained a cubic extension $U$ of $T$, then the dimension of $S$ over $T$ would be a multiple of three. On the other hand, if $S$ is obtained from $T$ by a tower of quadratic extensions, then the dimension of $S$ over $T$ is a power of two. Defining a configuration to be a finite collection $C$ of points, lines, and circles in a Euclidean plane, it can be stated, any point, line, or circle that can be constructed from the configuration $C$ is definable in a field obtained from the coefficients of all the objects in $C$ after taking a finite number of quadratic extensions, whereas trisection of angle $\angle C A B$ (an arbitrary angle in $C$ ) will basicallybe definable in a cubic extension of the field generated by the coordinates of $C, A, B$. Consider, based on these coordinates, three plane angles $\angle A B C, \angle B A C$ and $\angle A C B$ can be constructed to represent three different planes such that; the three plane angles add up to less than four right angles and any two of them add up to more than the third one [13]. These conditions can only be met in a non-Euclidean construction. For example, the three plane angles made from is osceles triangles of equal legs meet their vertices at a common endpoint. In classical Euclidean plane geometry, an angle is genetically defined in two dimensions $(x, y)$ with $x$ and $y$ as constructible points (not bas ed on coordinates), and not in three dimensions as implied from the Pierre Wantzel's general cubic equation of the angle trisection impossibility. From this discussion, it is evident that the presented proof of the impossibility statement obligates solving the angle trisection problem using methods made by bending the formal rules in the Euclidean rigor of constructions in a 2D geometry. Thus the presented proof of the impossibility, together with the revealed prohibited non Euclidean geometric solutions are geometrically incorrect and not valid in the jurisdiction of the traditional Greek's geometry.

\subsubsection{An interpreted Version of the Angle Trisection Impossibility Proof based on Trigonometry as illustrated in http://www.math.toronto.edu/rosent/Mat246Y/OLDPDF/week19.pdf [24]}

This section employ the use of algebra (trigonometry) to generate an algebraic proof of the angle trisection im possibility statement, based on the classification of rational numbers and surds. Consider: Suppose $C$ is a set of rational num bers, and suppose that $S$, is a field of all surds. Assuming that $C=S$, this section aims at showing that, one cannot trisect 
$60^{\circ}$ with only a straightedge and a compass. Consider the condition that; an angle is constructible for compass -ruler construction, if and only if its cosine is a constructible number as stated in section 1.3 . In this regard, $60^{\circ}$ angle is constructible, and the tris ection of $60^{\circ}$ would mean constructing an angle of $20^{\circ}$. A proof that $\cos 20^{\circ}$ is not a surd can be presented as follows:

Theorem 2: $A 60^{\circ}$ is not trisectable and therefore $\cos 20^{\circ}$ not constructible

To proof this theorem, consider the trigonometric identity;

$\cos (A+B)=\cos A \cos B-\sin A \sin B$

Working based on this identity;

$$
\begin{aligned}
& \cos 2 \emptyset=\cos ^{2} \emptyset-\sin ^{2} \emptyset=2 \cos ^{2} \emptyset-1 \quad \text { (The double-angle formula) } \\
& \cos 3 \emptyset=\cos (2 \emptyset+\emptyset) \\
& \cos 3 \emptyset=\cos 2 \emptyset \cos 2 \emptyset-\sin 2 \emptyset \sin \emptyset \\
& \cos 3 \emptyset=\left(2 \cos ^{2} \emptyset-1\right) \cos \emptyset-2 \sin \emptyset \cos \emptyset \sin \emptyset \\
& \cos 3 \emptyset=2 \cos ^{3} \emptyset-\cos \emptyset-2\left(1-\cos ^{2} \emptyset\right) \cos \emptyset \\
& \cos 3 \emptyset=4 \cos ^{3} \emptyset-3 \cos \emptyset \\
& \text { Taking }=20^{\circ}, \text { assume that; } \\
& \cos 3 \emptyset=\cos 60^{\circ}=1 / 2
\end{aligned}
$$

Therefore, $1 / 2=4 \cos ^{3} \emptyset-3 \cos \emptyset$

Equation (3) can be rewritten as;

$$
8\left(\cos 20^{\circ}\right)^{3}-6\left(\cos 20^{\circ}\right)-1=0
$$

Equivalently, taking $\cos 20^{\circ}=y$, equation (4) can further be rewritten as;

$$
8(y)^{3}-6(y)-1=0, \Rightarrow(2 y)^{3}-3(2 y)-1=0
$$

If we take $x=2 y$ and substitute for $2 y$ in equation (5), we get;

$$
x^{3}-3 x-1=0
$$

Clearly, if $\cos 20^{\circ}$ was in $C$, then $x$ satisfies the condition $x^{3}-3 x-1=0$

Claim: If $60^{\circ}$ was trisectible, there would be a constructible root of $x^{3}-3 x-1=0$

In algebraic view, it is clear that, if a cubic equation with rational coefficients has a constructible root, then it has a rational root. The goal here is to show that $x^{3}-3 x-1=0$ has no rational roots.

Suppose $x=a / b$ is a natural root. $a$ and $b$ are integers in their lowest form. Therefore,

$(a / b)^{3}-3(a) / b-1=0$

$a^{3} / b^{3}-3(a) / b-1=0$

$a^{3}-3 a b^{2}-b^{3}=0$

Consider;

If $p \backslash b$, then $p \backslash\left(3 a b^{2}-b^{3}\right)$, which implies $p \backslash a^{3}$, and hence $p \backslash a$

$p$ is prime, since for $a / b$ in its lowest terms, $p$ does not exist for the solutions \pm 1 .

If $q$ is prime and that $q \backslash a$, then $q \backslash\left(3 a b^{2}-b^{3}\right.$, and so $q \backslash b^{2}$. Therefore, $q \backslash b$. Thus, $a$ and $b$ are relatively prime, implying $a= \pm 1$. 


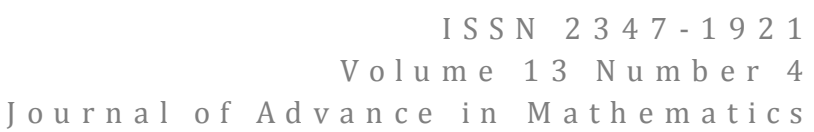

Taking $a, b= \pm 1$, implies $a / b= \pm 1$.

Therefore, if $20^{\circ}$ is constructible, then $x^{3}-3 x-1=0$, for $x=1$ or $x=-1$

Substituting for the two values of $X$ in the above equation;

$(1)^{3}-3(1)-1=-3$

$(-1)^{3}-3(-1)-1=1$

Based on these results, it can be stated that the equation $x^{3}-3 x-1=0$ is not algebraically reducible, as it was expected. From the presented two versions of the angle trisection impossibility proof, several facts as listed below, provide enough evidence to show that, the statement of angle trisection as an impossible problem for compassstraightedge construction has no geometrical cogency:

a) The presented algebraic proof of the angle trisection impossibility does not disprove a geometric solution or a concept aimed at solving the angle trisection problem, but, it is a proof of statement that the problem of angle trisection is generally unsolvable.

b) As observed from the presented discussions, each version in the proof settle at a three dimensional consideration that, the angle trisection solution corresponds to solving a certain cubic equation $\left(x^{3}-3 x-1=0\right)$, which is shown to be algebraicallyirreducible. This mode of approach turned the angle trisection problem from a plane geometric problem to a purely algebraic problem [6].

c) From section 1.3.2. equations $x^{3}-3 x-1=0$ is of the form $R^{3}$, a geometrical representation of an object in three dimensions. The genetic definition of the angle trisection problem is in two dimensions and not three dimensions. This show the ambiguityin the conjecture of relating cubic extension to angles based on towers. It is pellucid that any form of approach involving both cubic and quadratic equations is a solid geometric method, and not a Euclidean geometric problem [7]. It also has been shown that the angle trisection solution does not depend on the stated cubic equation, and it can be algebraically reduced to a quadratic equation [8]. Thus the angle trisection impossibility should not be geometrically acceptable.

d) The proof of angle trisection impossibility is based on trisection of a particular angle $60^{\circ}$, while according the geometrical definition of the problem as discussed in section 1.1 , the solution to the angle trisection problem should work for both a particular angle, and an arbitrary angle. It is entirely not clear how much more or less arbitraryis either $60^{\circ}$ or $30^{\circ}$ angle. This paper treats all the constructible angle multiples and submultiples of 15 as a specific angle and not an arbitrary case. The angle trisection impossibility statement is so obscure to depend on a specific constructible angle, to state a general conclusion that, if one cannot construct a certain angle, then one cannot solve the trisection of an arbitrary angle. This mode of solving the problem is purely arithmetical, which is geometrically prohibited. The solution to the angle trisection problem does not depend on the construction of a particular angle. It has been shown that it is geometrically possible to construct all whole number angles, and so is an angle of $20^{\circ}$, and the provided method did not solve the trisection of an arbitrary angle [1].

e) The algebraic condition specified in section 1.3; that a length is constructible if and only if it represents a constructible number show the geometrical limitations of these restrictions, since for example, it is geometricallypossible to cons truct lines of magnitude $\sqrt{2}, 2 \sqrt{2}$ and $\sqrt{5}$, and algebraic techniques have failed to conceive exact values for thes e irrational factors. Traditionally, Euclid worked with only positive integers. Negative numbers and surds were not accepted. The term irrationality did not exist. Thus, as it is geometrically possible to solve the square of listed surds, the angle trisection problem should be sought following the classical geometric rules and not based on algebra. Clearly, any other form of a solution not using the classical straightedge and compass is not geometrical.

From these interpretations, it is clear that the impos sibilitystatement for angle trisection problem should not be extended to resolution of angles, so that it is defined as; 'the angle trisection problem is an impossible problem of Euclidean geometry'. It is evidently shown how the presented algebraic proof is a sense of inability to solve the problem, and not an impossibility case as stated. This paper hope to resolve this ancient puzzle of angle trisection. Consider section 3.0.

\subsection{HYPOTHESIS}

As discussed from section 1.3 , it is pellucid that the problem of angle trisection concern serious misconceptions, and as a result, the proposed solutions are not geometrically correct. Section 1.2 , in a simple and brief approach dis cussed how the angle trisection problem should be soughtbased on the traditional mathematics frameworks of geometry. Accordingly, it is required that, for any given angle $\theta$, one be able to construct the fraction $\theta / 3$, under the set restriction of classical Greek's geometry. Consider figure (3); 


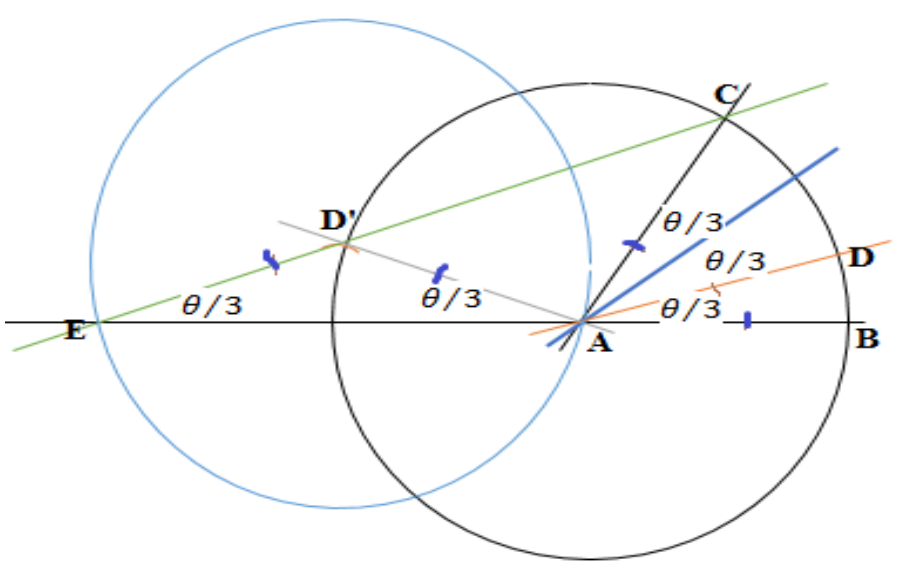

Fig 3: An arbitrary Angle Trisection Results

Figure (3) depicts the expected result for a correct trisection, an algorithm which should work for trisection of both an arbitrary angle, and a specific angle. Reasonably, for these type of results to be obtained, it is good for any construction to have a base to stem from. As such, $60^{\circ}$ angle form the base for all the constructible and trisectible angles multiples of 15 , their multiples and submultiples. Therefore, this work consider a certain set of angles, a range within which the trisection of both an arbitrary angle and a particular angle can be sought. This paper takes a quantum look at the ato $\mathrm{m}$ ic elemental quantities in a plane making the larger objects such as circles, lines, and angles, and the associated properties based on thes geometric quantities. In this case, all the angles in the range $0^{\circ}>\theta \leq 30^{\circ}$ are considered to form a subset of angles for the superset of all the angles in the intervals $0^{\circ}-360^{\circ}$ (both the whole number angles and the non-whole number angles), where the angle trisection problem could be sought at a good precision. This consideration is centered to the fact that, as it is shown in the trisection by Archimedes using a marked straight ed ge, it is possible to correctly trisect an acute angle. It is further considered that, trisection solution for any angle greater than $30^{\circ}$ is trisectible when the given angle is geometrically reduced to a value of an angle within the specified range as shown in section 4.0 and in section 5.0 .

\subsection{MATERIALS AND METHODS}

\subsection{Materials}

In this work, all the presented approaches are typically a compass-straightedge constructions, though some interactive computer software such as GeoGebra can be used, in respect to computer aided design approaches. In this work, GeoGebra 5.0 software as one of the interactive geometry software is used to bring the insight of computer aided design (CAD) methods, and for the visualization of the obtained results.

\subsection{Methods}

This chapter presents an elegant multistep procedure for solving the problem of angle trisection in antiquity. The initial part of this section provides a brief discussion to expose the rudimentaryidea and the logics employed in generating the proposed steps of construction. It then provides the general trisection solution, accompanied by an elementary proof to justify the correctness of the obtained results.

\subsubsection{Application of Set Theory in defining the Proposed Angles Trisection Solution}

The focus of this section is to reveal the geometrical outlook of the structure followed in deriving the assortment of angles for; $0^{\circ}>\theta \leq 30^{\circ}$, based on ideas from the set theory. Consider figure (4). 


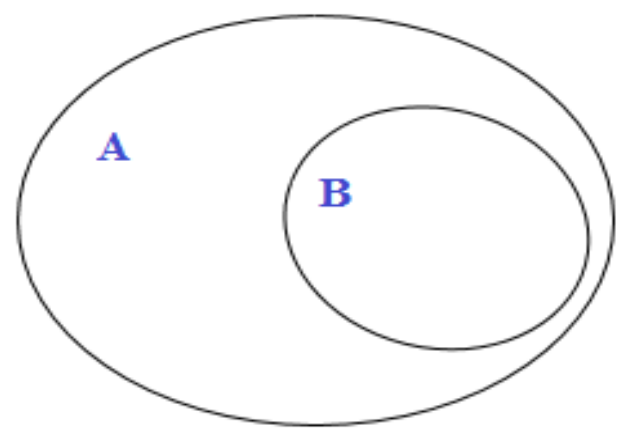

Fig 4: Geometrical Representation of some angle properties based on set theory, using a Venn diagram

In figure (4), region $A$ represents a superset of all angles on a Euclidean plane, while region $B$ is a subsetcontaining all the angles submultiples of the angle elements in the superset $A$. Specifically, an example to illustrate the relationship between the sets $A$ and $B$, is angles of magnitudes; $\pi / 4$, and $\pi / 6$. In this example, $\pi / 4$ is within region $A$, while $\pi / 6$ form the boundary, and a member in elements of the subset $B$. Consider that, the trisection results of $\pi / 4$ will appear in region $B$, as well as the trisection solution for $\pi / 6$. Again, both the angle $\pi / 8$ and its trisection solution lie in region $B$. Conversely, trisecting an angle in the subset $B$ would imply the possibility of trisecting its multiple angles in the superset $A$. In this case, if we are in a position to trisect any given case in the subset $B$, then any other angle in the superset $A$ is also trisectible. The aim of this paper is to provide a geometrical approach to verify the claim that, any given angle in the superset $A$, can geometrically be trisected from the subset $B$, if and only if any angle element within the subset $B$ is trisectible. That is: if an angle $\theta \in B$ is trisectible in the range $0^{\circ}>\theta \leq 30^{\circ}$, then since $\theta \in A$, any other angle element in $A$ is geometrically trisectible, because $B \subset A$. However, for a specific angle $\beta$ such that either, $\beta \in A$ or $\beta \in B$, and that if $\beta \in A$ and $\beta \notin B$, then the angle trisection problem cannot be sought by trisecting the angle $\beta$, since in this case, any form of solution should work for any angle lying either in the subset $B$, or in the supers et $A$. Consider the following theorem:

Theorem 3: It is geometrically possible to trisect an arbitrary angle under the specified restrictions of classical geometry.

Under this claim, consider the following construction steps, meant to elaborate on the discussion in s ection 3.2.1. Figure (5) depicts the construction results.

\subsubsection{Trisection of an Arbitrary Angle using only a compass and a straightedge (ruler)}

1. For a given $\angle C A B$, use the radius defined by ray $\overline{A B}$ (the baseline) to construct a $30^{\circ}$ angle $D A B$.

2. If the given $\angle C A B$ lie within $\angle D A B$ defined by the range $0^{\circ}>\theta \leq 30^{\circ}$, label $C^{\prime}$ the point of intersection between curve $B D$ and ray $\overline{A C}$. However, if $\angle C A B$ is outside the region $\angle D A B$, reduce geometrically $\angle C A B$ to its submultiple angle $\angle C^{\prime} A B$, such that $\angle C^{\prime} A B$ lie somewhere in $\angle D A B$, using a compass and straightedge (ruler).

3. Assuming that $\angle C A B$ lie at, or within $\angle D A B$, Join points $C^{\prime}$ and $B$ using a straight line, and construct the bis ection of ray $\overline{A B}$ at a point $E$.

4. Using circles of radius $\overline{B E}$ and the ray $\overline{B A}$, trisect the chord $\overline{C^{\prime} B}$ at points $F$ and $G$ as shown in figure (5). See trisection of $\overline{C^{\prime} B}$ in annex-1.

5. Draw a straight line from the center of the fourth circle (point $E^{\prime}$ ) of trisection on ray $\overline{B A}$, through point $F$ to cut the curve $B C^{\prime}$ at point $H$ as shown below.

6. Again, draw a straight line through points $A$ and $G$, to cut curve $B C^{\prime}$ at point $I$ as shown in figure (5).

7. With the compass placed at point $B$, mark an arc of radius $H I$ along curve $B C^{\prime}$ at a point $/ .3 \angle J A B=\angle C^{\prime} A B$. 


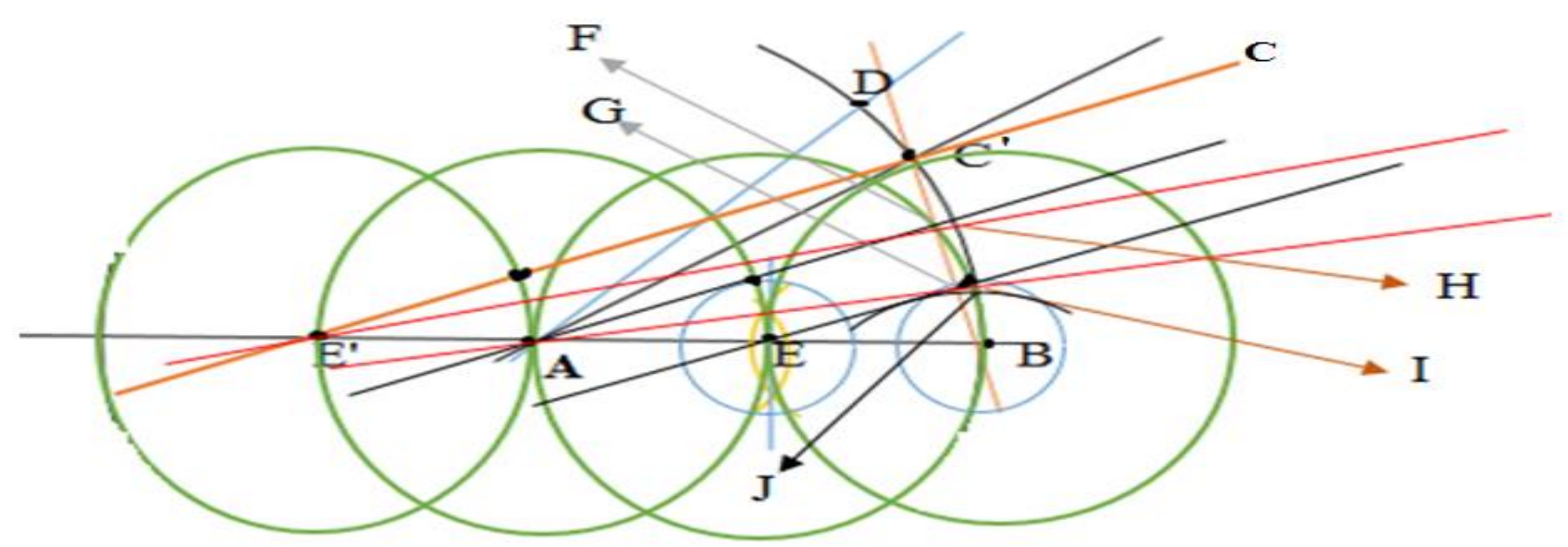

Fig 5: Trisection Results for $\angle C^{\prime} A B$

The above procedure present a claim that, $\angle J A B=1 / 3\left(\angle C^{\prime} A B\right)$. To verify this statement, consider the following sections of proof.

\subsubsection{Geometrical Proof that $\angle J A B=1 / 3\left(\angle C^{\prime} A B\right)$}

The aim of this section is to expose the accuracy of the assertion that $\angle J A B=1 / 3\left(\angle C^{\prime} A B\right)$. The initial part of this proof concerns the application of the compass equivalence theorem, to show the correctness of the found results, bas ed on some properties of points and lines in the realm of circles. The second part of this proof involves the analytical interpretation of some geometric properties inherent from the construction results, and their relationships, to show geometrical precision of the proposed algorithm.

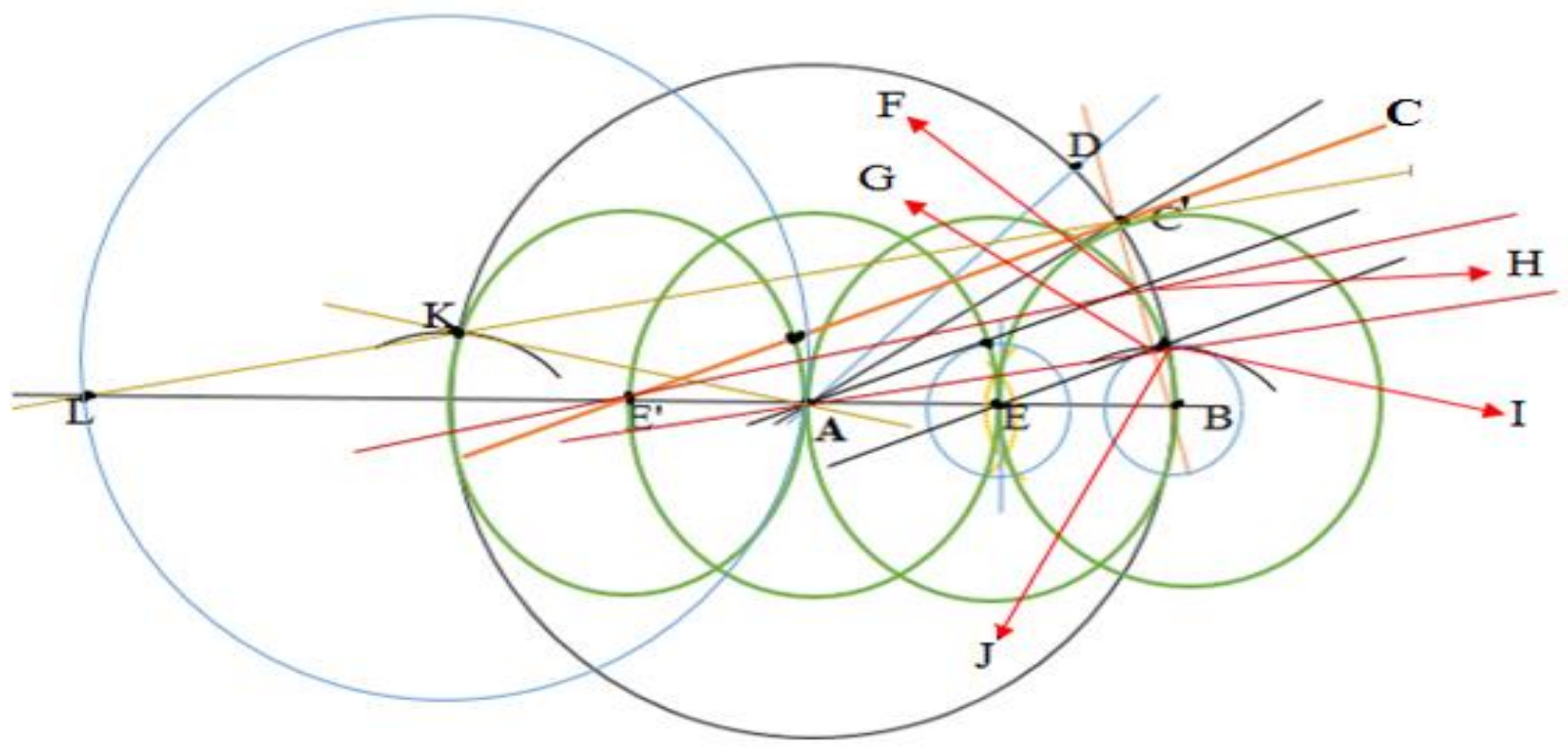

Fig 6: An application of the compass equivalence theorem to verify that $\overline{L K} \cong \overline{K A} \cong \overline{A B}$

\subsection{Geometrical Transformation of point $I$ to point $K$}

\section{Proof:}

This section of proof is depended on the compass equivalence theorem to justifythe claim that, if point $K$ is an image of point $I$ (through a geometric transformation), and a straight line is drown through points $C^{\prime}$ and $K$ to produce point $L$ ( $L$ is 
the point of intersection between the base line and the line through points $C^{\prime}$ and $K$, implying the three points are collinear), it can be shown that $\overline{L K} \cong \overline{K A} \cong \overline{A B} \cong r$ and that $\angle J A B \cong \angle L A K \cong \angle A L K \cong 1 / 3\left(\angle C^{\prime} A B\right)$. Considering figure (6), point $K$ is a reflection of point $J$, about center $A$ through a certain angle $90^{\circ}-2 \theta$ as shown in 3.2.2.1.2. In figure (6), the circle in blue, centered at point $K$, pass through the vertices $L$ and $A$. Point $A$ is the center of the initial circle used in mapping point $I$ to point $K$. This implies that $\overline{L K} \cong \overline{K A} \cong \overline{A B} \cong r$, where $r$ radius of the circle centered at point $A$. The compass equivalence theorem as provided in proposition $I I$ in Book $I$ of Euclid's Elements states "anyconstruction via 'fixed' compass maybe attained with a collapsing compass. That is; it is possible to construct circles of equal radius centered at any point on a plane" [2], [12], [16]. Thus assuming an arbitrarypoint $K$ to be center of a circle (the circle in blue curvature), the compass equivalence theorem helps confirm that $\overline{L K} \cong \overline{K A} \cong \overline{A B} \cong r$, by constructing a circle that passes through points $L$ and $A$ (with point $A$ as the center of the initial circle (the circle in black curvature)) as required.

\subsection{An analytical proof that $\angle J A B \cong 1 / 3\left(\angle C^{\prime} A B\right)$}

In section 3.2.2.1.1 it is shown that, $\overline{L K} \cong \overline{K A} \cong \overline{A B} \cong r$, with $r$ as the radius of the circle in black curvature. This part of proof is aimed at showing that $\angle J A B \cong \angle L A K \cong \angle A L K \cong 1 / 3\left(\angle C^{\prime} A B\right)$, based on the property; $\overline{L K} \cong \overline{K A} \cong \overline{A B} \cong r$. Consider figure (7). Point $B^{\prime}$ is a reflection of point $B$ about point $A$. Taking $\angle J A B \cong \angle B^{\prime} A K=\theta$, and that point $K$ is a reflection of point $J$ (proven), then $\angle K A L=\angle K L A=\theta$, (Base angles of is osceles triangle $A K L$ since $\overline{L K} \cong \overline{K A} \cong \overline{A B} \cong r^{r}$ ).

This implythat;

Triangle $K A B^{\prime} \equiv J A B$, (Congruence property $\left.S A S\right)$.

Again, $\angle A K C^{\prime}=2 \theta$ (by property, an exterior angle of a triangle is equal to the sum of the opposite interior angles).

Also $\angle A C^{\prime} K=2 \theta$, (Base angles of isosceles triangle $K A C^{\prime}$ ).

It follows that $\angle K A C^{\prime}=180^{\circ}-4 \theta$

Consider; $\angle B^{\prime} A K+\angle K A C^{\prime}+\angle C^{\prime} A B=180^{\circ}$ (Sum of angles on a straight line add up to $180^{\circ}$ ). Making $\angle C^{\prime} A B$ the subject; $\angle C^{\prime} A B=180^{\circ}-\left(\angle B^{\prime} A K+\angle K A C^{\prime}\right)$

From equations (7) and (9) we have $\angle B^{\prime} A K=\theta$ and $\angle K A C^{\prime}=180^{\circ}-4 \theta$ respectively. Substituting for angles $\angle B^{\prime} A K$ and $\angle K A C^{\prime}$ in equation (10) we have;

$\angle C^{\prime} A B=180^{\circ}-\left(\theta+\left(180^{\circ}-4 \theta\right)\right)$

The equation reduces to $\angle C^{\prime} A B=-\theta+4 \theta=3 \theta$

Equation (11) imply that $\angle C^{\prime} A B=3 \angle B^{\prime} A K$, which can be rewritten as;

$1 / 3\left(\angle C^{\prime} A B\right)=\angle B^{\prime} A K$

Thus $1 / 3\left(\angle C^{\prime} A B\right) \cong \angle J A B \cong \angle L A K \cong \angle A L K$, since $\angle J A B \cong \angle B^{\prime} A K$, hence the claim proven.

Further, from equation (8), $1 / 2\left(\angle K A C^{\prime}\right)=90^{\circ}-2 \theta$, is the angle through which point $I$ is mapped onto point $K$, about point $A$. This observation can be stated classicallyin theorem form as;

Theorem 4:"The classical trisection solution for a given angle is always, either; within a set of angles in the range $0^{\circ}>\theta \leq 30^{\circ}$, or it is a multiple, or a submultiple of an angle in the configuration $0^{\circ}>\theta \leq 30^{\circ}$, , as sought using the described method. 


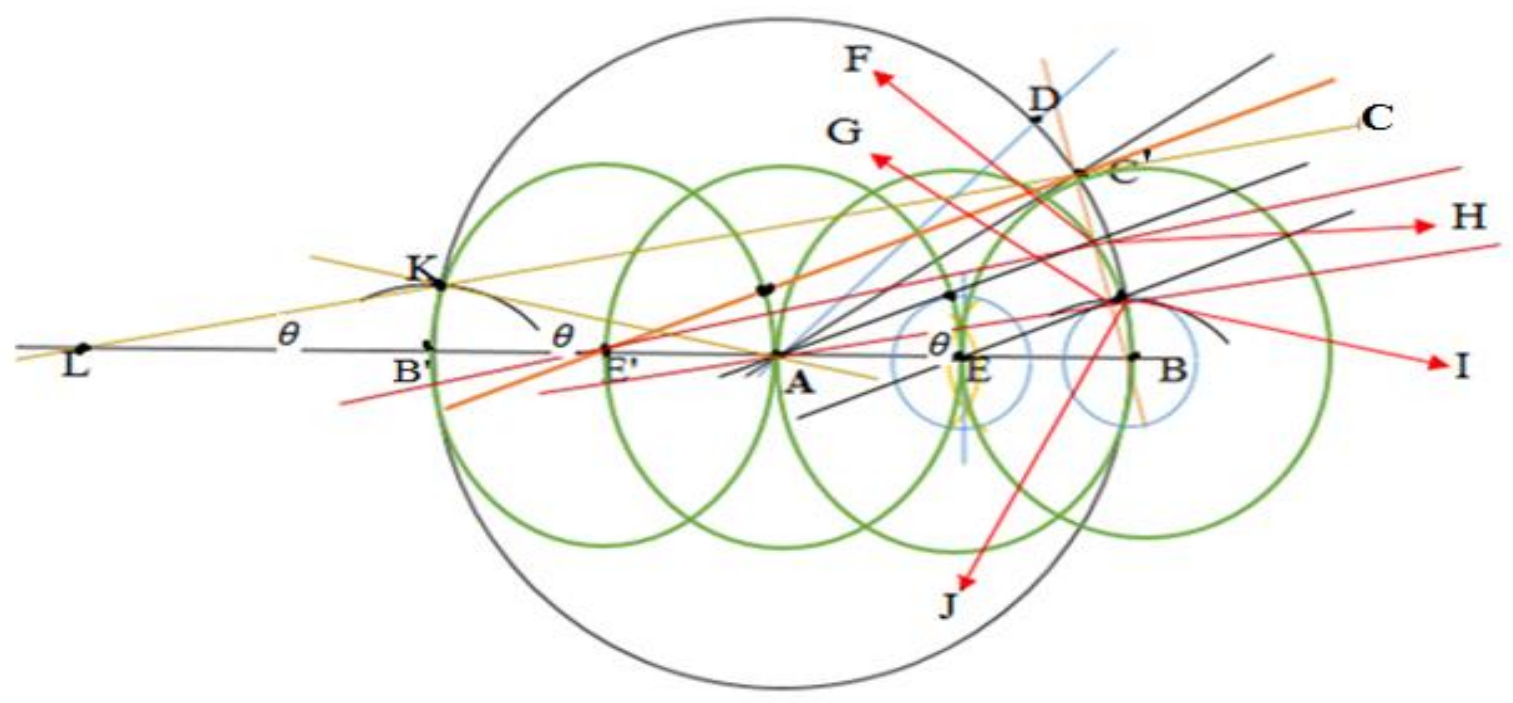

Fig 7: General Classical Geometric Proof of Angle Trisection

\subsection{Justification of the Method}

The proposed algorithm is justified by performing the trisection of some angles within the range $0^{\circ}>\theta \leq 30^{\circ}$, inclusive of the $30^{\circ}$, and also, in the trisection of $45^{\circ}$ angle, with the $45^{\circ}$ representing all angles in the supers et $A$, as described in section 3.2.1. In all the presented construction methodologies, GeoGebra software is used for results visualization.

\subsubsection{Geometrical Trisection of $30^{\circ}$ using Compass and Straightedge (Ruler) only}

The following steps of construction would help trisect an angle of size $30^{\circ}$. The method works for both the compass-ruler construction, and when performed using a computer software.

1. For given two points $A$ and $B$, use radius $\overline{A B}$ to construct a $30^{\circ}$ angle $C A B$ as shown in figure (8).

2. Join points $C$ and $B$ using a straight line, and construct the bisection of ray $\overline{A B}$ at a point $D$.

3. Using circles of radius $\overline{B D}$ and the ray $\overline{B A}$, trisect the chord $\overline{C B}$ at points $E$ and $F$ as shown in figure (8). See trisection of $C B$ in annex-1.

4. Draw a straight line from the center of the fourth circle (point $D^{\prime}$ ) of trisection, on ray $\overline{B A}$, through point $E$ to cut the curve $B C$ at point $G$ as shown.

5. Again, draw a straight line through points $A$ and $F$, to cut curve $B C$ at point $H$ as shown in figure (8).

6. With the compass placed at point $B$, mark an arc of radius $G H$ along curve $B C$ at a point $I . \angle I A B=10^{\circ}$.

Figure (8) is the construction results obtained. As seen from the algebra view, $\angle C A B=30^{\circ}$ has been trisected. 


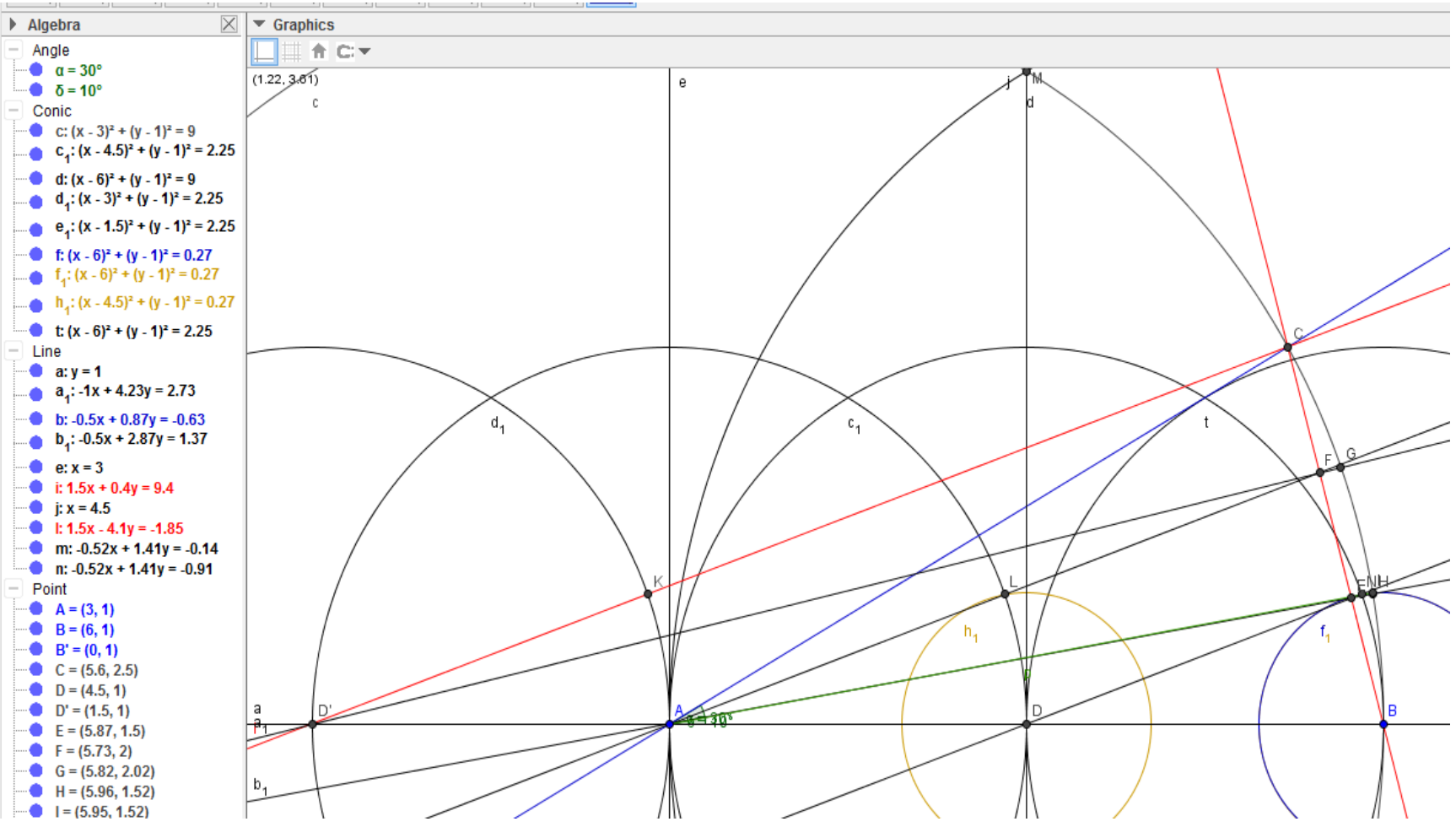

Fig 8: Trisection of $30^{\circ}$ angle using GeoGebra software

\section{Proof}

This proof presents a general overview for all the ensuing forms of verifications to these types of construction. As s tated before, the compass equivalence theorem could provide a reasonable justification that the trisection results are geometrically precise. Figure (9) is the geometric proof that $3 \angle I A B=\angle C A B$ after performing the following construction procedure using GeoGebra software:

1. For given two points $A$ and $B$, use radius $\overline{A B}$ to constructa $30^{\circ}$ angle $C A B$ as shown in figure (9).

2. Join points $C$ and $B$ using a straight line, and construct the bisection of ray $\overline{A B}$ at a point $D$.

3. Using circles of radius $\overline{B D}$ and the ray $\overline{B A}$, trisect the chord $\overline{C B}$ at points $E$ and $F$ as shown in figure (9).

4. Draw a straight line from the center of the fourth circle (point $D^{\prime}$ ) of trisection, on ray $\overline{B A}$, through point $E$ to cut the curve $B C$ at point $G$ as shown.

5. Again, draw a straight line through points $A$ and $F$, to cut curve $B C$ at point $H$ as shown in figure (9).

6. With the compass placed at point $B$, mark an arc of radius $G H$ along curve $B C$ at a point $I$.

7. Reflect point $B$ about point $A$ to produce $B^{J}$ as shown.

8. Using the radius $B I$, place the compass at point $B^{\prime}$ and make an arc to cut circle centered at $A$, at a point $I^{\prime}$.

9. Draw a straight line through points $C$ and $I^{\prime}$, to cut line $A B^{\prime}$ externally at a point $I$.

10. Use radius $I^{\prime}$ to construct a circle centered at $I^{\prime}$ as shown in figure (9). 

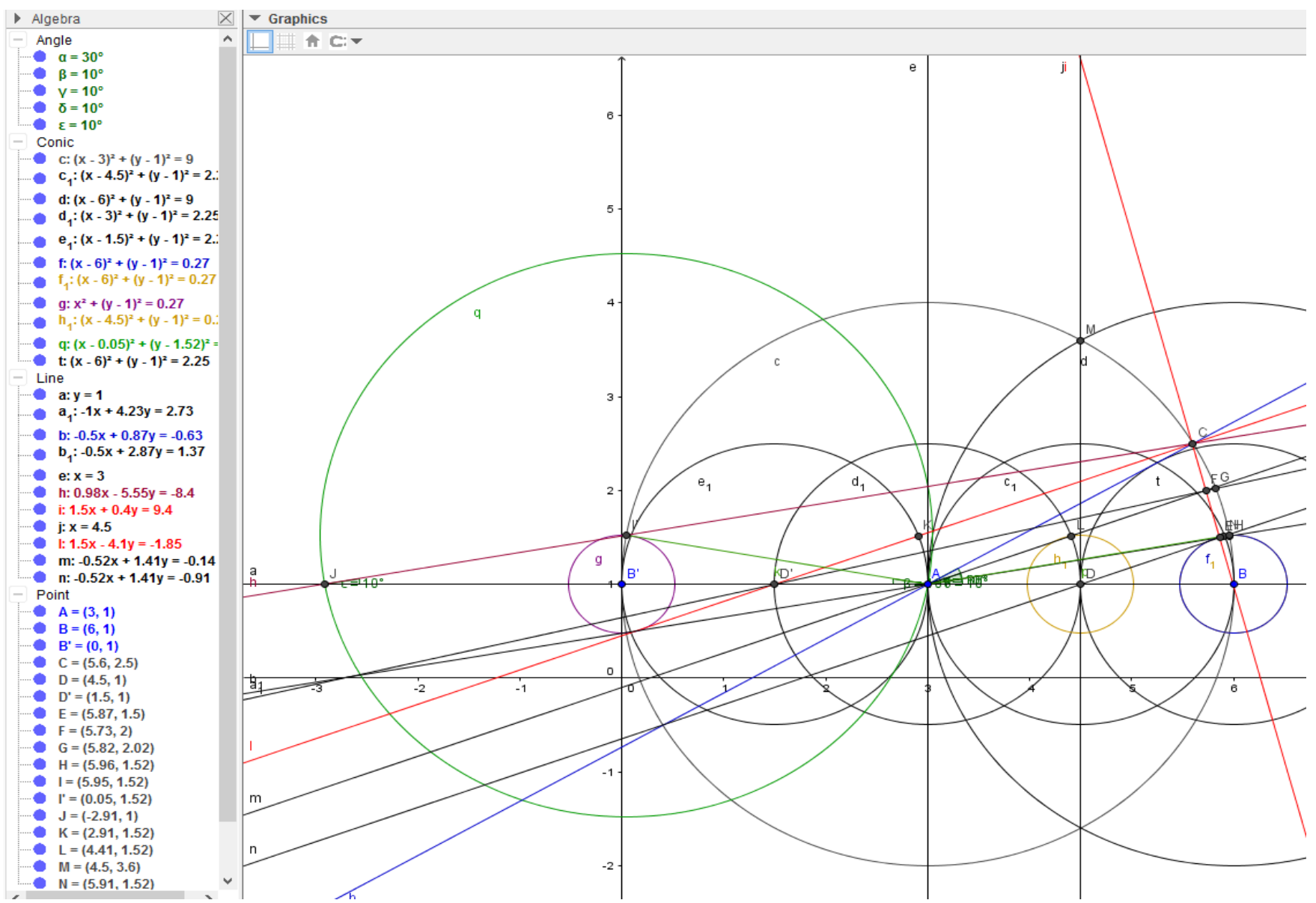

Fig 9: Proof of $30^{\circ}$ angle trisection results, an application of the compass equivalence theorem

\subsubsection{Geometrical Trisection of $17.1^{\circ}$ angle Using GeoGebra Software}

Figure (10) is the results obtained after carrying out the following steps of construction using GeoGebra software.

1. For the given $\angle Z A B \cong 17.1^{\circ}$, use radius $\overline{A B}$ to cons truct a circular curve that cuts ray $Z A$ at a point $C$.

2. Join points $C$ and $B$ using a straight line, and construct the bisection of ray $\overline{A B}$ at a point $D$.

3. Using circles of radius $\overline{B D}$ and the ray $\overline{B A}$, trisect the chord $\overline{C B}$ at points $E$ and $F$ as shown in figure (10). See trisection example on annex-1.

4. Draw a straight line from the center of the fourth circle (point $D^{\prime}$ ) of trisection, on ray $\overline{B A}$, through point $F^{\prime}$ to cut the curve $B C$ at point $G$ as shown.

5. Again, draw a straight line through points $A$ and $E$, to cut curve $B C$ at point $H$ as shown in figure (10).

6. With the compass placed at point $B$, mark an arc of radius $G H$ along curve $B C$ at a point $I . \angle I A B=5.7^{\circ}$, as depicted in the algebra view window. 

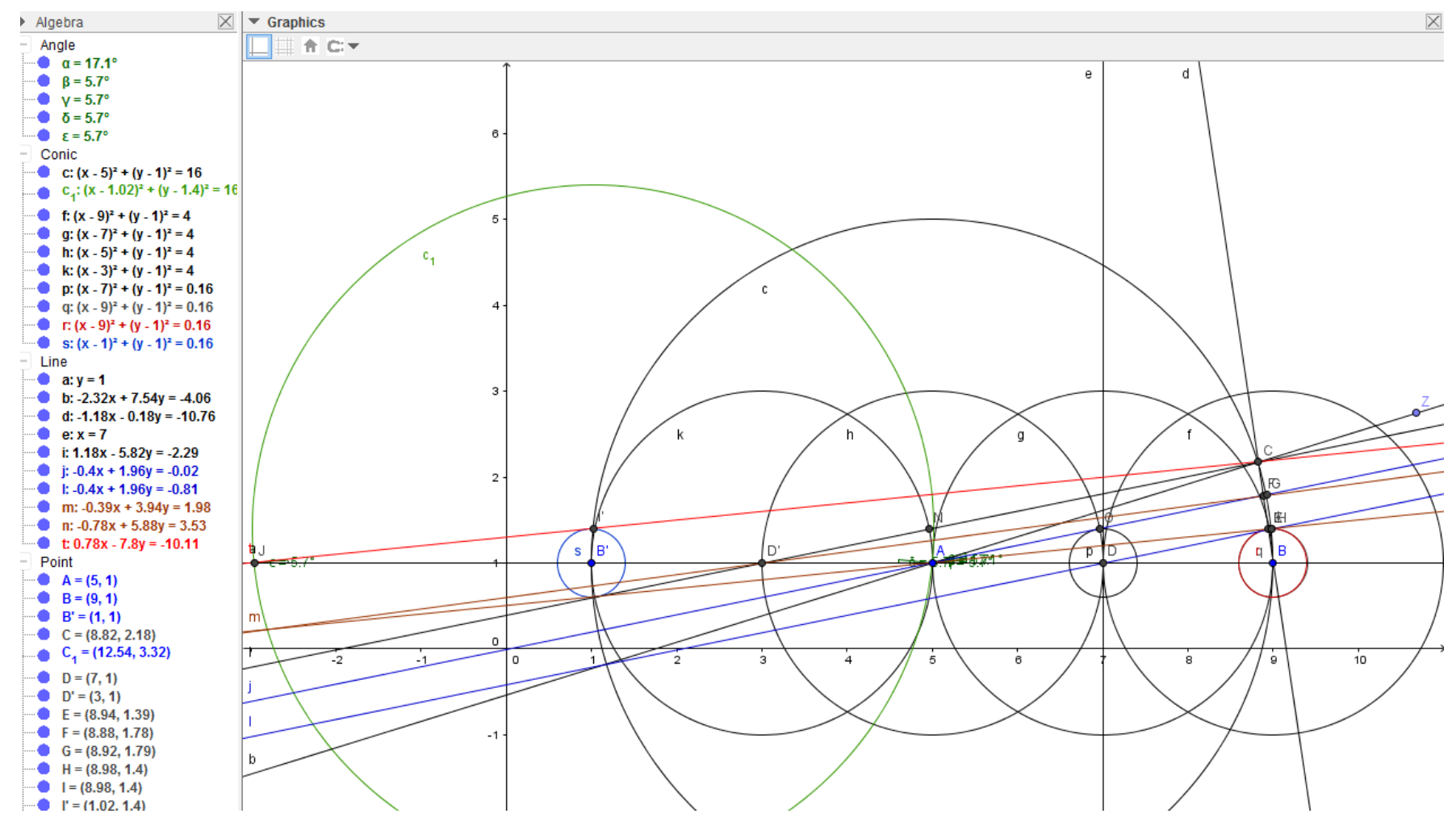

Fig 10: Geometrical trisection results for $17.1^{\circ}$ angle using the GeoGebra software

\subsubsection{Geometrical Trisection of $4.629^{\circ}$ angle Using the GeoGebra software}

This section presents a construction for trisecting an angle of 4.629 using the GeoGebra software, to show the correctness of the proposed method, at an accuracy of $4 \mathrm{~s} . \mathrm{f}$. Consider the following construction:

1. For the given $\angle Z A B \cong 4.629^{\circ}$, use radius $\overline{A B}$ to construct a circular curve to cut ray $Z A$ at a point $C$.

2. Join points $C$ and $B$ using a straight line, and construct the bisection of ray $\overline{A B}$ at a point $D$.

3. Using circles of radius $\overline{B D}$ and the ray $\overline{B A}$, trisect the chord $\overline{C B}$ at points $E$ and $F$ as shown in figure (11). See trisection example on annex-1.

4. Draw a straight line from the center of the fourth circle (point $D^{\prime}$ ) of trisection, on ray $\overline{B A}$, through point $F$ to cut the curve $B C$ at point $G$ as shown.

5. Again, draw a straight line through points $A$ and $E$, to cut curve $B C$ at point $H$ as shown in figure (11).

6. With the compass placed at point $B$, mark an arc of radius $G H$ along curve $B C$ at a point $I . \angle I A B=1.543^{\circ}$, as depicted in the algebra view window. 


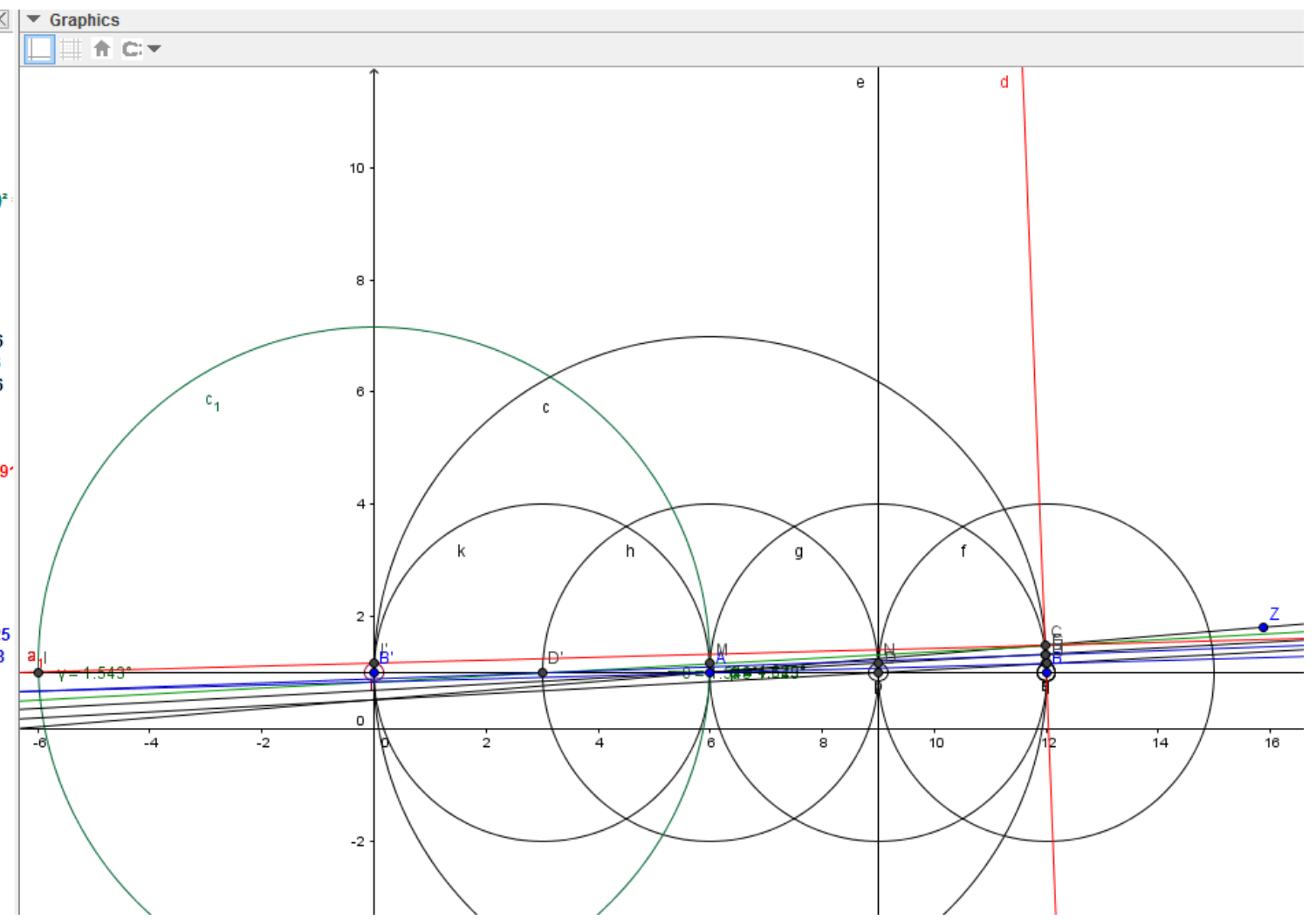

Fig 11: A Geometrical Trisection illustrative Proof for $4.629^{\circ}$ angle

\subsection{4 trisection of 45 angle to justfy validity of the proposed range $0^{\circ}>\theta \leq 30^{\circ}$ of angles for trisetion solutions}

This section applies the proposed algorithm in trisection of $45^{\circ}$ angle, to justify the theorem that the trisection of both a particular angle and an arbitrary angle for compass and straightedge (ruler) construction lie in the array $0^{\circ}>\theta \leq 30^{\circ}$ as defined earlier. Consider the following construction and the obtained results:

1. Given that $\angle C A B \cong 45^{\circ}$, use radius $\overline{A B}$ to construct a circular curve through point ray $C$.

2. Join points $C$ and $B$ using a straight line, and construct the bisection of ray $\overline{A B}$ at a point $D$.

3. Using circles of radius $\overline{B D}$ and the ray $\overline{B A}$, trisect the chord $\overline{C B}$ at points $E$ and $F$ as shown in figure (12). See trisection example on annex-1.

4. Draw a straight line from the center of the fourth circle (point $D^{\prime}$ ) of trisection, on ray $\overline{B A}$, through point $F$ to cut the curve $B C$ at point $G$ as shown.

5. Again, draw a straight line through points $A$ and $E$, to cut curve $B C$ at point $H$ as shown in figure (12).

6. With the compass placed at point $B$, mark an arc of radius $G H$ along curve $B C$ at a point $I . \angle I A B=15.01^{\circ}$, while its reflection at point $B^{\prime}$ is $15^{\circ}$, as depicted in the algebra view window in figure (12). 


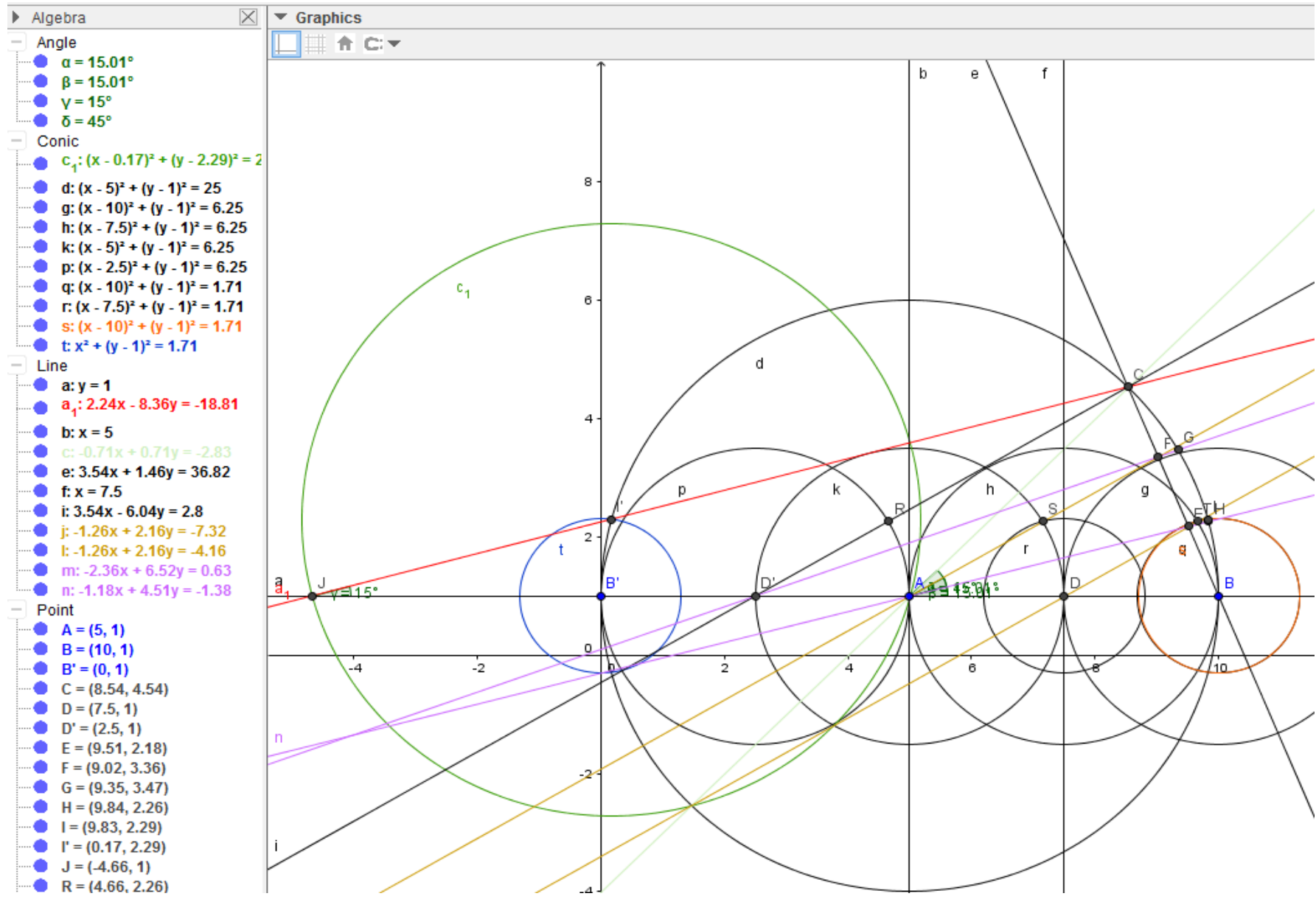

Fig 12: Trisection of 45 angle using GeoGebra software

\subsubsection{Geometrical Trisection of $22.5^{\circ}$ angle to confirm that all the angles greater than $30^{\circ}$ angle are trisectible within the range; $0^{\circ}>\theta \leq 30^{\circ}$}

This construction begins by the bisection of a $45^{\circ}$ degree angle to produce $\angle C A B=22.5^{\circ}$, and the above construction steps repeated to produce figure (13):

1. Given that $\angle Z A B \cong 45^{\circ}$, constructa bisection of $\angle Z A B$ to get an angle of $22.5^{\circ}$.

2. Using radius $\overline{A B}$ to construct a circular curve to cut the bisection at a point $C$.

3. Join points $C$ and $B$ using a straight line, and construct the bisection of ray $\overline{A B}$ at a point $D$.

4. Using circles of radius $\overline{B D}$ and the ray $\overline{B A}$, trisect the chord $\overline{C B}$ at points $E$ and $F$ as shown in figure (13). See trisection example on annex-1.

5. Draw a straight line from the center of the fourth circle (point $D^{\prime}$ ) of trisection, on ray $\overline{B A}$, through point $F$ to cut the curve $B C$ at point $G$ as shown.

6. Again, draw a straight line through points $A$ and $E$, to cut curve $B C$ at point $H$ as shown in figure (13).

7. With the compass placed at point $B$, mark an arc of radius $G H$ along curve $B C$ at a point $I . \angle I A B=7.5^{\circ}$, as depicted in the algebra view window of the GeoGebra software. 


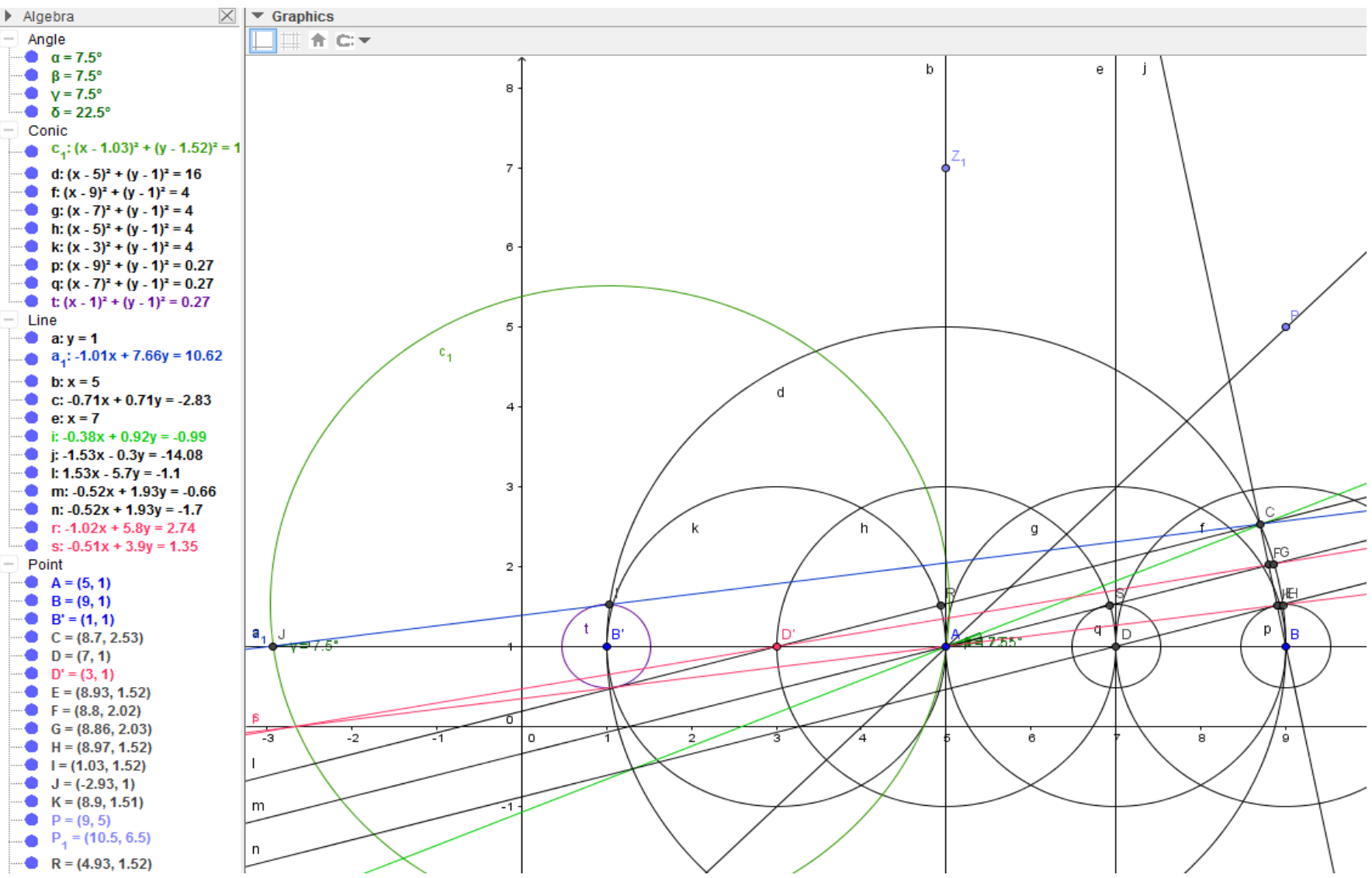

Fig 13: Trisection of 22.5 angle to confirm that 45 angle is only trisectible within the limits $0^{\circ}>\theta \leq 30^{\circ}$

\subsection{APPLICATION}

\subsection{Trisection of Obtuse Angles}

Generally, it is required that, a correct angle trisection method should work for any given angle, regardless of its size. As stated earlier, $45^{\circ}$ is only precisely trisectible within the limit $0^{\circ}>\theta \leq 30^{\circ}$. The goal of this section is to provide an illustrative construction of trisecting an obtuse angle. The most influential trisection solution of an obtuse angle was presented by Archimedes (287-217 B.C.), [4], [11]. The construction involves using a marked straightedge and a compass, mechanically to construct an angle one third the size of a given angle [5], [15]. As demonstrated in his proof, it is difficult to verify the trisection of an obtuse angle, than it is to proof the trisection results of an acute angle. In th is work, based on the stated limits of the angle trisection solution, the tris ection proof presented is considered to generalize for the trisection of any angle. Clearly, if one is able to provably trisect an angle within the range $0^{\circ}>\theta \leq 30^{\circ}$ and that all angle in this configuration are trisectible, so is any required trisection proof within the same array, and thus any other given angle is trisectible. Thus the following example of tris ecting $165.96^{\circ}$ angle is an illustration of how the construction should be performed and not a proof. The results in figure (14) were obtained using GeoGebra software for the follows steps of construction:

\subsection{Geometrical Trisection of $\angle C A B \cong 165.96^{\circ}$}

Angle $C A B \cong 165.96^{\circ}$ was obtained randomlyby locating points $A, B$ and $C$ on a plane, and drawing the two rays $\overline{C A}$ and $\overline{B A}$ to a common point $A$. To trisect this angle, its size has to be geometrically reduced to an angle $F A B$ of value within the range $0^{\circ}>\theta \leq 30^{\circ}$ (defined by $\angle U A B$ ), as depicted in figure (14); (where $\angle U A B$ has to be constructed before fractioning the given angle). In this case, $\angle F A B \cong 1 / 8(\angle F A B)$. That is; $\angle F A B$ octsect $\angle C A B$. The trisection of $\angle F A B$ can be performed as follows;

1. Join points $F$ and $B$ using a straight line, and construct the bisection of ray $\overline{A B}$ at a point $G$. 
2. Using circles of radius $\overline{B G}$ and the ray $\overline{B A}$, trisect the chord $\overline{F B}$ at points $L$ and $K$ as shown in figure (14). See trisection example on annex-1.

3. Draw a straight line through points $A$ and $K$, to cut curve $B F$ at point $M$ as shown in figure (14).

4. Again draw a straight line from the center of the fourth circle (point $G^{\prime}$ ) of trisection, on ray $\overline{B A}$, through point $L$ to cut the curve $B F$ at point $N$ as shown.

5. With the compass placed at point $B$, mark eight(8) equal intervals using radius $\overline{M N}$ along curve $B F$. For this example, this operation was performed from point $B^{\prime}$ to produce $\angle R A B^{\prime}$, which was then reflected to the opposite side on the curve as $\angle S A B$.

As seen from the algebra window of the GeoGebra software, $\angle S A B \cong 55.32^{\circ}$, while $\angle C A B \cong 165.96^{\circ}$. Clearly, $165.96^{\circ} / 3=55.32^{\circ}$.
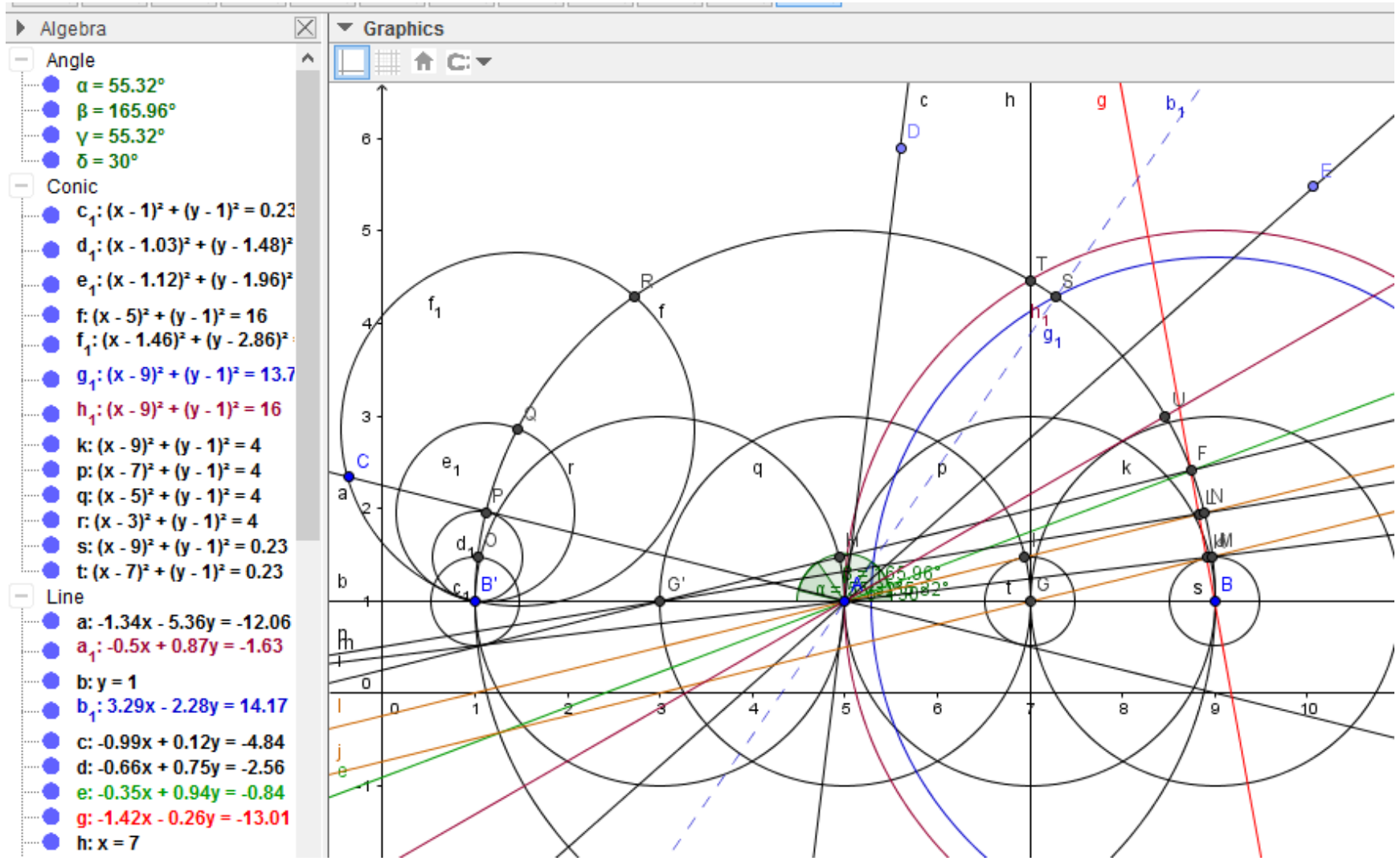

Fig 14: Trisection Results of Obtuse Angle $\angle C A B \cong 165.96^{\circ}$

Considering the construction results in figure (14), $\angle U A B=30^{\circ}$ (which has to be constructed before the trisection process is executed), is a bisection of $\angle T A B=60^{\circ}$. Therefore, $\angle F A B$ obey the condition $0^{\circ}>\theta \leq 30^{\circ}$. The process of trisecting $\angle C A B$ by stroking the curve $N M$ a number of times along the circumference $B S B^{\prime}$ on the plane does not violate any classical geometric rule. The same application is employed in the construction of regular polyg ons, such as an hexagon [20].

\subsection{RESULTS AND DISCUSSION}

The geometrical representation of results in this work is from figure (6) to figure (17). Figure (6) and figure (7) presents the general trisection results, based on the proposed algorithm. These diagrams illustrate both the geometrical and the analytical interpretation of the obtained results, to ratify the exactness of the methods, respectively. As stated earlier, it is entirely not clear to what degree of arbitrariness is an angle of $30^{\circ}$. In this proof, the $30^{\circ}$ angle is taken to be both a specific angle, and an arbitrary angle. The $30^{\circ}$ is thus regarded as the maximum limit of the proposed angle trisection solution, within which, any given angle inclusive of the $30^{\circ}$ angle is trisectible. Figure (8) and figure (9) present the 
verified solutions to the trisection of $30^{\circ}$ angle. Further, figure (10) and figure (11), provide clear results of trisecting angles of $17.1^{\circ}$ and $4.629^{\circ}$ respectively, to show that the revealed method works for any given angle within the specified spectrum. Figure (12) depicts the general trisection results of all the angles outside the scale; $0^{\circ}>\theta \leq 30^{\circ}$, by trisecting an angle of magnitude $45^{\circ}$. As observed from figure (12), the size of $\angle I A B \cong 15.01^{\circ}$, while that of the reflected $\angle I^{\prime} A B^{\prime} \cong 15^{\circ}$. These variations are not observed from the trisection results of all the other cases within the scale of $0^{\circ}>\theta \leq 30^{\circ}$, where the obtained results demonstrate the exactness of the method. This implythe validity of theorem 4 , in its consideration towards a solution to the old age classical problem of angle trisection. From the obtained results (figure (6) to figure (13)), the purpose of the circle in green and radius $I^{\prime}$ (in all cases point $I^{\prime}$ is an image of $I$ ) is to show the application of the compass equivalence theorem in determining the accuracy of the provided methods. The goal of trisecting an angle of $22.5^{\circ}$ was to show that the trisection of the $45^{\circ}$ angle could, precisely be sought by trisecting an angle, which is a submultiple of $45^{\circ}$, and lying in the interval $0^{\circ}>\theta \leq 30^{\circ}$. Therefore, to trisect $45^{\circ}$, one has to duplicate the curve subtending $\angle I A B$ as in figure (13), along the curve $B C B^{\prime}$. As conferred in section $3.2 .1^{\circ}$, an angle of $45^{\circ}$ would lie in the superset $A$, while its trisection solution is found by working out the proposed construction on its submultiple angle within the subset $B$. Thus it is geometrically possible to trisect all angles, if the proposed method is performed under the range $0^{\circ}>\theta \leq 30^{\circ}$. Figure (14) provide a clear illustration to elaborate on the trisection of an obtuse angle. To this point, the demonstrated results involve the trisection of all angle in the intervals $0^{\circ}>\theta \leq 180^{\circ}$. The classical geometric trisection of any angle greater in magnitude than an angle of $180^{\circ}$ (that is: $\theta>180^{\circ}$ ) can geometrically be obtained by subtracting the reflex angle $\alpha^{\circ}$ from $360^{\circ}$ and performing the proposed construction for the difference $360^{\circ}-\alpha^{\circ}=\emptyset^{\circ}$. The trisection results of the angle $\alpha^{\circ}$ would then be given by $120^{\circ}-\left(\emptyset^{\circ} / 3\right)=\alpha^{\circ} / 3$ as described in [8], among the other many simple ways of solving such a construction.

\section{Classical geometric trisection of the Special Anqles $\beta=360^{\circ}$ and $\beta=0^{\circ}$}

Theorem 5: It is geometricallypossible to trisect an angle of magnitude $360^{\circ}$

As stated earlier, the term area or volume of modern world did not apply in classical geometry. However, study results show that Euclid employed the arithmetic operation of multiplication when determining the content (Area in modern world) of similar objects such as triangles and rectangles, by use of the term commeasurable. In classical geo metry, angles are also considered as magnitudes. Employing the same technique of proportions of magnitudes of similar kind, this paper consider the ratio $360^{\circ} / 120^{\circ}$ to justify the assertion that all angles are trisectible within intervals $0^{\circ}>\theta \leq 30^{\circ}$. Consider, $360^{\circ}$ angle is not definable between a union of two rays as most angles would appear, and so is an angle of magnitude $0^{\circ}$. This view completely eliminate the classical trisection of the angle $0^{\circ}$ (trisection angle of $0^{\circ}$ goes to infinity, since it is only defined by a single axis), due to consideration that in classical geometry, the magnitude $0^{\circ}$ did not exist. However, $360^{\circ}$ is an angle multiple of $30^{\circ}$, the limit of the proposed range, when the $360^{\circ}$ is defined in a circle on a plane. Based on proportionality, $360^{\circ} / 30^{\circ}=12$, where in this case, the result 12 is taken to be a magnitude of two ratios. This implies, the trisection of $360^{\circ}$ would be obtained by classical tris ection of $30^{\circ}$, and stroking 12 equal intervals of curve subtending trisection angle of $30^{\circ}$ at a point $O$, along the circle circumference. Alternatively, one can generate an angle of value $60^{\circ}$ using the provided algorithm, where the supplementary angle would be of magnitude $120^{\circ}$. Thus the singular angle $360^{\circ}$ is geometrically trisectible.

\subsection{Accuracy of the Proposed Method}

The accuracy of the generated method could well be determined using an interactive computer software, since the traditional Greek's tools of geometry produce an accuracy of up to tenths, though with an application of a Vanier scale, higher precisions than tenths can be obtained. Figures (15) and (16) provide a comparative view of the results obtained when the proposed method is applied for the trisection of $60^{\circ}$ at different reference angles $\left(30^{\circ}\right.$ and $\left.15^{\circ}\right)$ respectively.

\subsection{Trisection of $30^{\circ}$ angle to confirm the trisection of $60^{\circ}$ angle at $5 \mathrm{dp}$ Accuracy}

Consider figure (15) obtained after carrying out the following steps of cons truction using GeoGebra software:

1. For given two points $A$ and $B$, use radius $\overline{A B}$ to construct $30^{\circ}$ angle $D A B$ as shown in figure (15). 
2. Join points $D$ and $B$ using a straight line, and construct the bisection of ray $\overline{A B}$ at a point $E$.

3. Using circles of radius $\overline{B E}$ and the ray $\overline{B A}$, trisect the chord $\overline{D B}$ at points $F$ and $G$ as shown in figure (15). See trisection example on annex-1.

4. Draw a straight line from the center of the fourth circle (point $E^{\prime}$ ) of trisection, on ray $\overline{B A}$, through point $G$ to cut the curve $B C$ at point $H$ as shown.

5. Again, draw a straight line through points $A$ and $F$, to cut curve $B C$ at point $I$ as shown in figure (15).

6. With the compass placed at point $B^{\prime}$, mark an arc of radius $H I$ along curve $B C$ at a point $J . \angle J A B=10.00105^{\circ}$.

From figure (15), $\angle C A B=60^{\circ}$, and $\angle D A B=30^{\circ} . \angle D A B=30^{\circ}$ has been fractioned into three mean proportions of size; $\angle J A B=10.00105^{\circ}$. From these results, point $I^{\prime}$ is a reflection of point $I$. As seen from the algebra window, $\angle J^{\prime} A B^{\prime}=10.00105^{\circ}, \angle K A B^{\prime}=20.0021^{\circ}$, and $\angle K L B^{\prime}=19.99895^{\circ}$.
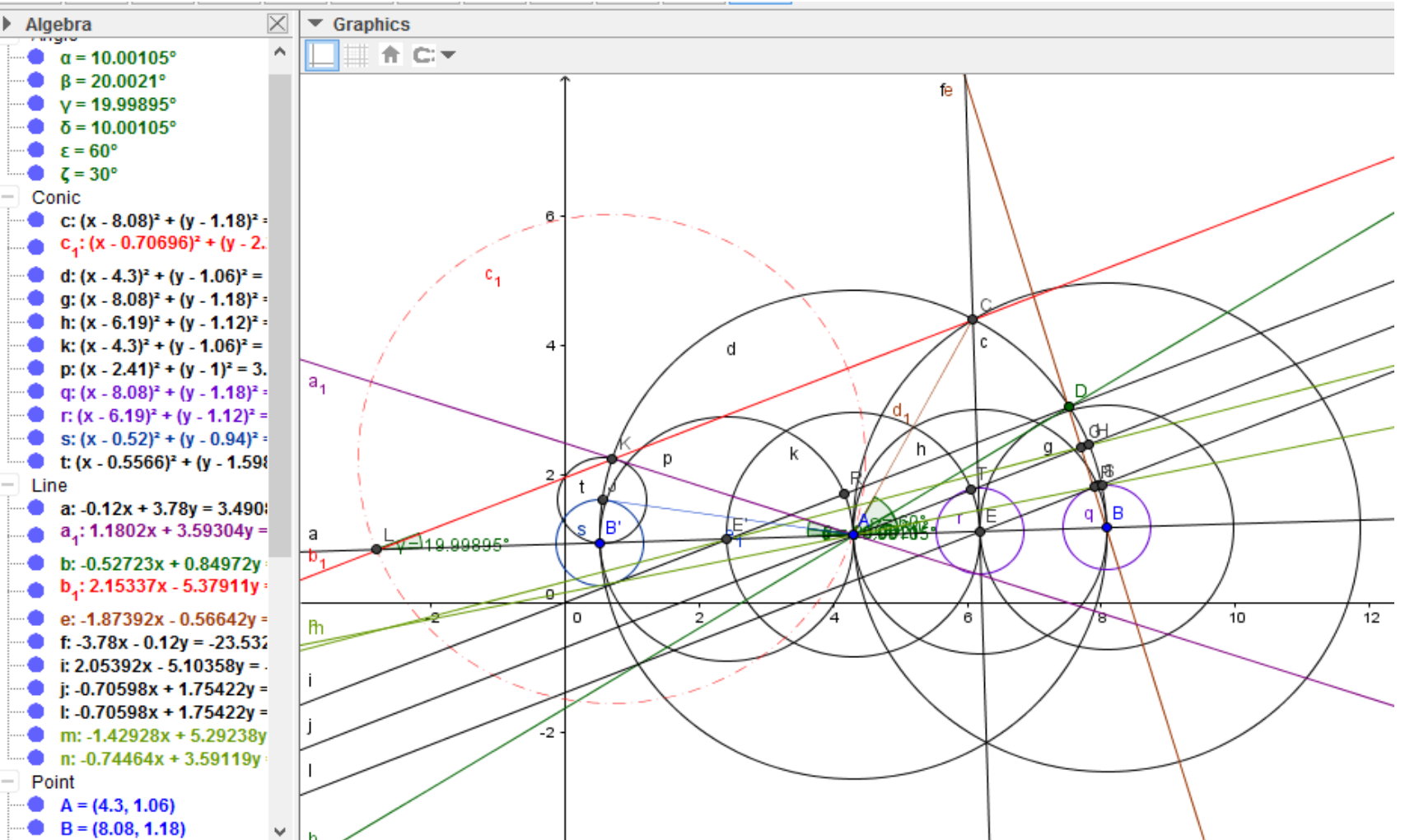

Fig 15: Trisection of $60^{\circ}$ angle to an accuracy of 5 decimal places

\subsection{Trisection of $15^{\circ}$ angle to confirm the trisection of $60^{\circ}$ angle at $5 d p$ Accuracy}

Consider the follows procedure and the obtained results:

1. For given two points $A$ and $B$, use radius $\overline{A B}$ to construct a $15^{\circ}$ angle $E A B$ as shown in figure (16).

2. Join points $E$ and $B$ using a straight line, and construct the bisection of ray $\overline{A B}$ at a point $F$.

3. Using circles of radius $\overline{B F}$ and the ray $\overline{B A}$, trisect the chord $\overline{E B}$ at points $G$ and $H$ as shown in figure (16). See trisection example on annex-1.

4. Draw a straight line from the center of the fourth circle (point $F^{\prime}$ ) of trisection, on ray $\overline{B A}$, through point $H$ to cut the curve $B C$ at point $I$ as shown. 
5. Again, draw a straight line through points $A$ and $G$, to cut curve $B C$ at point $/$ as shown in figure (16).

6. With the compass placed at point $B$, mark an arc of radius $I J$ along curve $B C$ at a point $K$. $\angle K A B=5.00003^{\circ}$ as depicted in figure (16). $\angle K^{\prime} A B^{\prime}=5.00003^{\circ}$ is a reflection of $\angle K A B=5.00003^{\circ}$.

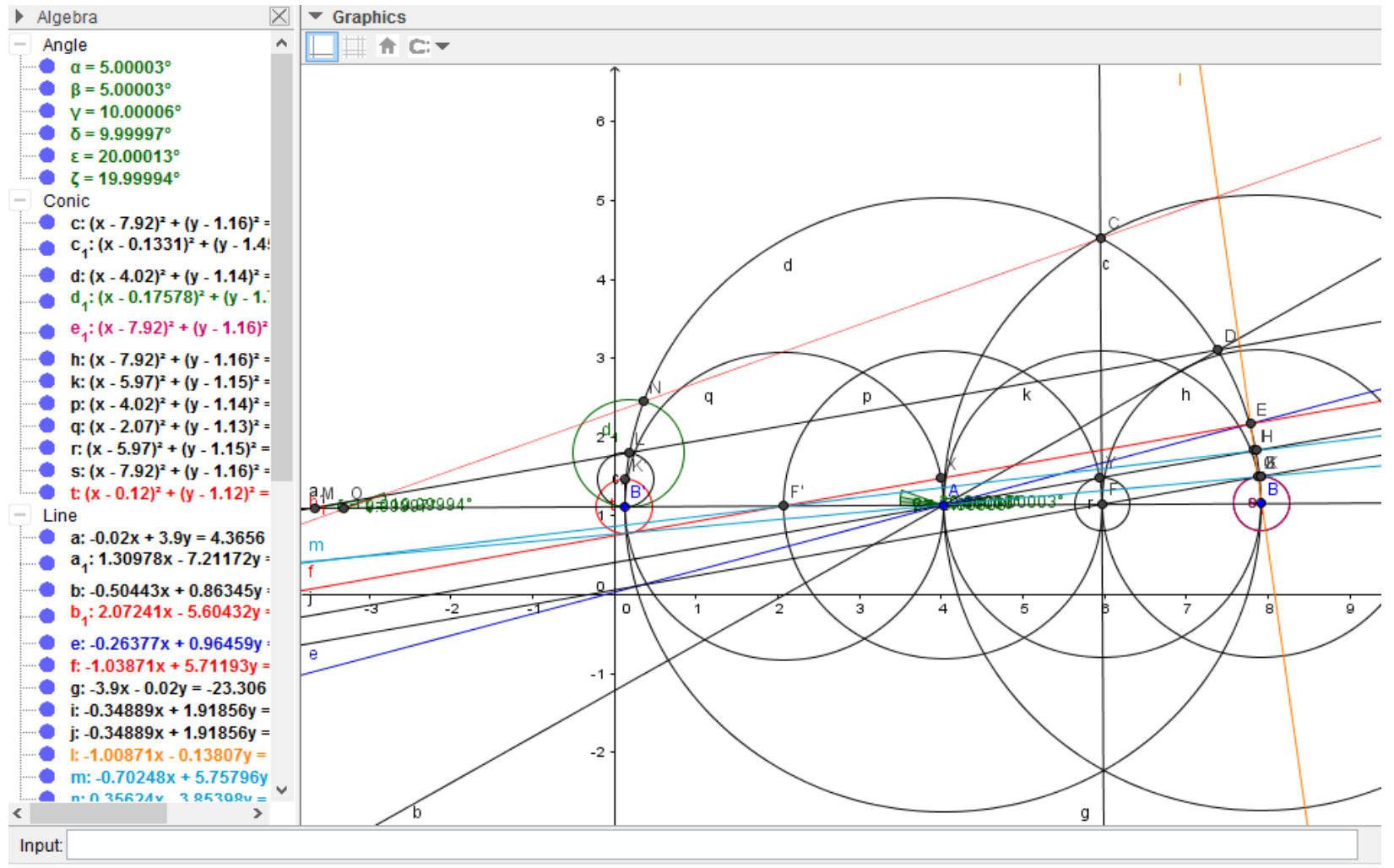

Fig 16: Trisection of $15^{\circ}$ angle to confirm the accuracy of trisecting $60^{\circ}$ angle to $5 d p$

Consider figures (15) and (16). Both results show the trisection of $60^{\circ}$ at two reference angles of magnitude $30^{\circ}$ and $15^{\circ}$ respectively. From the figures, it is observed that, the smaller the size of the angle to be trisected, the higher the accuracy. That is; from figure (15), two of the angles are $20.0021^{\circ}$ and $19.99895^{\circ}$, while the respective angles in figure (16) have sizes $20.00013^{\circ}$ and $19.99994^{\circ}$. Thus as the size of the angle to be trisected has to be reduced to a reasonable value within the proposed range. However, all the obtained results are logicallyprecise, since both the $30^{\circ}$ angle and the $15^{\circ}$ angle lie within the limits $0^{\circ}>\theta \leq 30^{\circ}$. According to this paper, this is the highest accuracy and possibly the exact solution to be discovered under the set restrictions of solving the angle trisection problem. Moreover, it should be considered that, Euclid did not work with the real numbers in classical geometry, instead, he employed us e of magnitudes. All classical proofs were inherent from the constructions. As demonstrated in this paper, the obtained results are geometrically exact, and therefore the angle trisection problem is solved. The use of the open source GeoGebra (5.0) in this paper was preferred because of its good geometry and algebra toolboxes, which create the environment for algebraic visualization of the geometricallygenerated results.

\subsection{CONCLUSION}

Throughout the presented discussion in this paper, an attem pt has been made to proof that the angle trisection problem of the ancient Greek mathematics is geometrically solvable, by providing an elegant multis tep method governed by the classical rules of Euclidean geometry. The analogs involved concerns the quantum look of some elemental quantities (elemental lengths and curves), purposely to inspect the geometrical relationships of different quantities on a gi ven plane. It has also been shown that the angle trisection impossibility statement, together with the presented non-Euclidean solutions [14], [17], [18], has no geometric rationality. It can therefore be concluded that, the angle trisection impossibility statement is not geometrically valid, and that the angle tris ection problem is resolvable following the provided method, for the configuration $0^{\circ}>\theta \leq 30^{\circ}$ and the condition demonstrated in Annex-1. The proposed proof works for both compass-straightedge (ruler) construction, and the computer aided design approaches. Trisection of any angle in the range $0^{\circ}>\theta \leq 30^{\circ}$ infer the trisection of any angle (whole number angles and non-whole number angles defined 


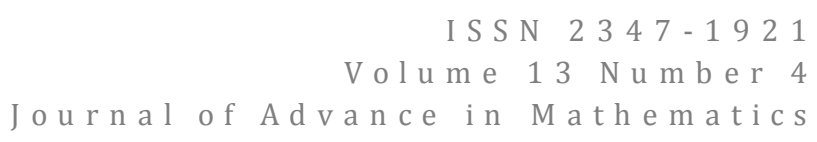

between any two rays) as illustrated in sections 4.1 , and 5.0 , thus the sethypothesis achieved. However, as dis cus sed earlier, any other form of solution involving the use of algebra (trigonometry) is not a classical geometric solution. Moreover, the use of a computer software in this works was purely for results visualization, and not as part of the provided proofs. The geometrical analysis of the result did not employthe use of coordinates (as done in analytical geometry), and this as pectbrings in the traditional Euclid's ways of analys is, based on proportions between quantities of sim ilar kinds. The use of the interactive computer software pose some challenge in that, they have some limiting factors bas ed on the software development logic. Therefore, with this present theorem developed under a multistep construction procedure, the approximate results displayed using the GeoGebra software cannotbe final for the discretion of the propos ed m ethods. This consideration is due to the fact that in geometric constructions, no construction can produce an exact measurement (only reasonablycorrect logical flow make sense). Genetically, the proposed method is rationally, geometricallycorrect. It reveals one of the most elegant provable compass and straightedge construction of the $21^{\text {st }}$ century.

\section{ACKNOWLEDGMENTS}

The authors of this paper gratefully acknowledge the support by all friends, and reviewers who took part in evaluating the developed methods. Sincerely thanks to God for his strength and teaching of the patience in taking up this work.

\subsection{REFERENCES}

1. K. M. Alex. 2017 The Possibility of Angle trisection (A Compass Straightedge Construction), Journal of Mathematics and System Science, Vol.7, (January, 25, 2017), P. 25-42. doi: 10.17265/2159-5291/2017.01.003.

2. W.S. Anglin. 1994. Mathematics: A Concise Historyand Philosophy, Springer-Verlag, New York, (1994), pp. 75-80.

3. C. B. Boyer. 1991. A History of Mathematics, 2nd Ed., John Wiley \& Sons, Inc, (1991), p.64.

4. H.Dorrie. 1965.100 Great Problems of Elementary Mathematics, Dover Publications, New York, (1965), P. 173.

5. J. Castellanos. 1994-2007. What is Non-Euclidean Geometry?, $<$ https://www.cs.unm .edu/ joel/NonEuclid/noneuclidean.html>, (1994)-(2007).

6. Burton, M. David. 1999. A History of Mathematics: An Introduction, 4th Ed., McGraw-Hill, p. 116, (1999).

7. R. Descartes. 1664. La Géométrie, C. Angot, Paris, (1664) (first published in 1637).

8. H. Florentino Ĺatortue. 2017. The Solution to the Impossible Problem, Library of Congress Control Number: 2017905297, NY, Printed in the United States of America, (2017).

9. C.A.Hart and D. D. Feldman. 1912, 2013. Plane and Solid Geometry, American Book Company, Chicago, (1912), (2013), p.341-342.

10. Dudley, U. 1983. What to do when the trisection comes. The Mathematical Intelligencer 5, (1), (1983), p.21.

11. Dudley, Underwood. 1994. The Trisectors, The Mathematical Association of America.

12. R. Fitzpatrick. 1883-1885. Euclid's Elements of Geometry, from Euclidis Elementa, edidit et Latine interpretatus est I.L. Heiberg, in aedibus, B.G. Teubneri.

13. C.A.Hart and Daniel D Feldman. 1912, 2013. Plane and Solid Geometry, American Book Company, Chicago, (1912), (2013), p.96, p.171.

14. Henderson, W David et al. 2005. Experiencing Geometry/Euclidean and Non-Euclidean with History ( $3^{\text {rd }}$ Ed), Pearson/ Prentice-Hall, ISBN 0-13-143748-8.

15. Thomas, I. 1939, 1941. Selections Illustrating the History of Greek Mathematics, William Heinemann, London, and Harvard University Press, Cambridge, MA.

16. Stillwell, John. 1989. Mathematics and Its History, Springer-Verlag, New York.

17. Faber, Richard L. 1983. Foundations of Euclidean and Non-Euclidean Geometry, New York, Marcel Dekker, Inc. ISBN 0-8247-1748-1.

18. J. Marshall. 2014. What Are Euclidean and Non-Euclidean Geometry?, <http://www.quickanddirtytips.com/education/math/what-are-euclidean-and-non-euclidean-geometry>, October 17, (2014).

19. Burton, D. M. 1999. A History of Mathematics: An Introduction, 4th Ed., McGraw-Hill, (1999), p. 116.

20. S. Mustonen. 2013. Simple Constructions' of Regularn-sided Polygons at Any Given Accuracy, University of Helsinki, April, 30, (2013).

21. Hartshorne, Robin. 2000. Geometry: Euclid and Beyond, Springer-Verlag, (2000), p. 10.

22. University of Toronto. 1997. The Three Impossible Constructions of Geometry, <mathnet@math.toronto.edu>, 14 Aug, (1997).

23. P.L. Wantzel. 1837. Recherches sur les moyens de reconnaitre si un problème de géométrie peut se rés oudre avec la règle et le compass. Journal de Mathematiques pures et appliques, (1837), Vol. 2, pp.366-372. 
24. K.M. Alex. 2017. Short Review on Classical Construction of Whole Number Angles and The Angle Trisection Problem (A Compass-Straightedge Construction), Indian Journal of Science and Technology, (2017), Accepted for Pub licatin.

\section{ANNEXES}

\section{Annex-1 Classical Trisection of the Chord $B C$ or $B C^{\prime}$ of all Constructions}

The following steps generalize for the geometric trisection of the line segment $B C$ or $B C^{\prime}$, joining points $B$ and $C$ or points $B$ and $C^{\prime}$ (or any other chord of similar kind) in all provided constructions. Initially, one is require to construct the bisection of the ray $\overline{A B}$ at a point, say $D$, and reflect the point $D$ about point $A$ to get point $D^{\prime}$ (the governing trisection condition for Alex kimuya's Trisection theorem in $30^{\circ}$ configuration).

1. Construct the first circle using radius $B D$ and center $B$.

2. Without adjusting the span of the compass, construct the second, third, and the fourth circles using centers $D, A$, and $D^{\prime}$ respectively.

3. Join points $C$ or $C^{\prime}$ (if using $C^{\prime}$ ) to pint $D^{\prime}$ using a straight line, and lebel $E$, the point of intersection between line $C D$ and the fourth circle.

4. With the compass placed at point $B$, mark point $F$ on the second circle using radius $E A$ as shown in figure 17 ..

5. Again, with the compass at point $D$, mark a point $G$ on the third circle using chord $E A$ as illus trated below.

6. Draw a straight line through points $A$ and $G$ to cut the chord $\overline{C B}$ at a point $H$.

7. Draw a straight line through points $D$ and $F$ to cut chord $\overline{C B}$ at a point $I$.

The segment $\overline{C B}$ is geometrically trisected. Lines $\overline{A H}$ and $\overline{D I}$ are parallel (according to Thale's theorem). One can use comparisons to verify the equality of the three line proportions, $B I, I H$, and $H C$, to each other.

Consider the figure 17:
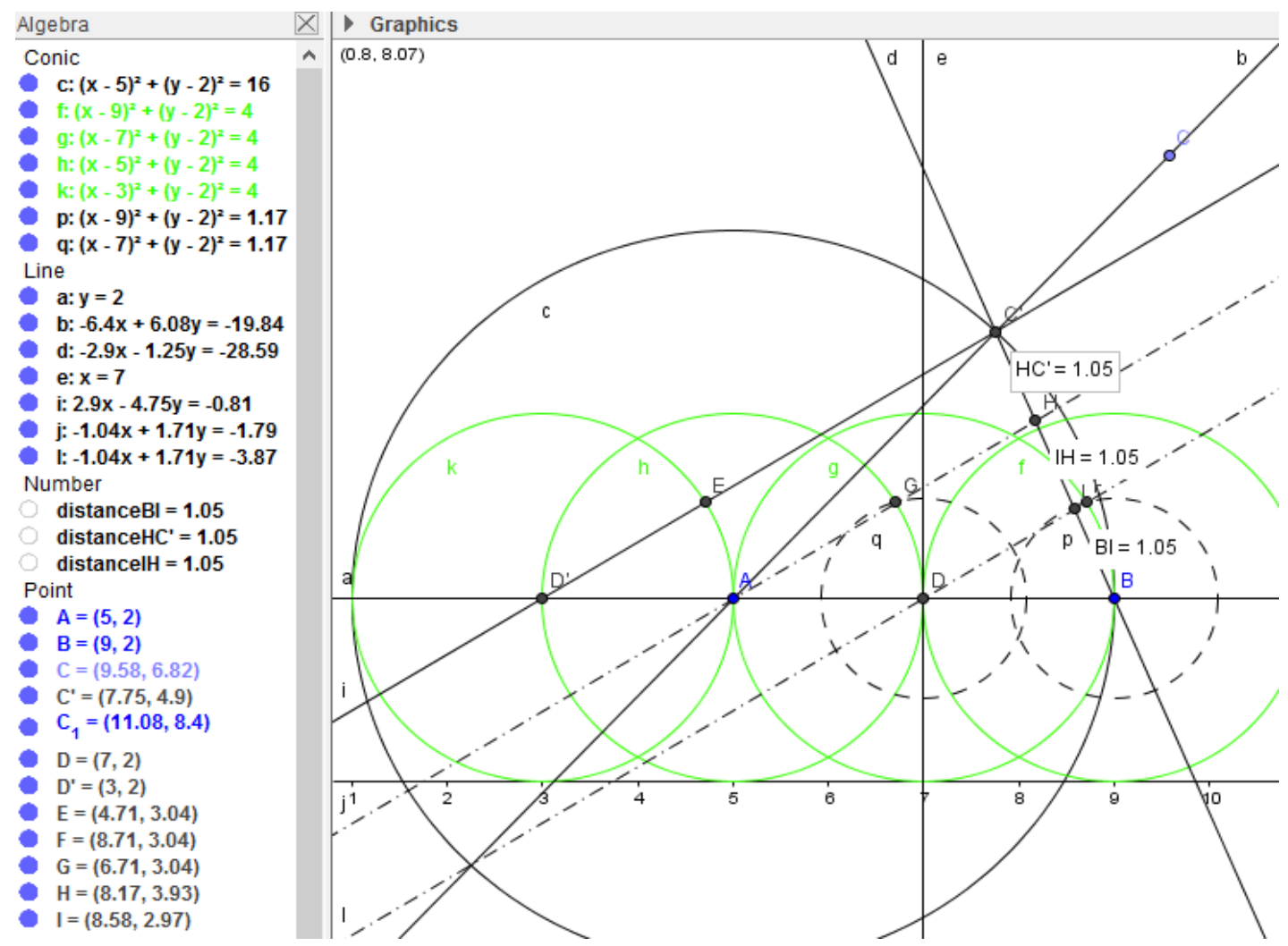

Fig 17: How to Trisect the chord $C^{\prime} B$ 


\section{AUTHOR' BIOGRAPHY}

Kimuya M Alex holds Bachelor of Science degree in Physics from Meru University of Science and Technology (2016). In between his undergraduate course, Alex worked as a freelance computational scientist in areas of mathematics, physic $s$, and machine learning. He is currently working as a lecturer, Meru University of Science and Technology. His research interests include:Applied Sciences (Mathematics and Physics), Geometry, Computer Vision, Machine Learning, and Big Data Analytics. He has a good amplitude in mathematics, particularly in graphical methods (Computer Graphics).

\section{РНОТО}

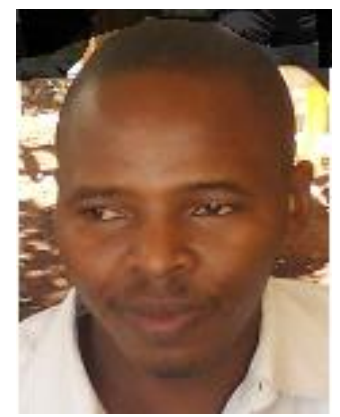

